\title{
Dynamical Analysis of SIR Epidemic Models with Distributed Delay
}

\author{
Wencai Zhao, ${ }^{1}$ Tongqian Zhang, ${ }^{1}$ Zhengbo Chang, ${ }^{1}$ Xinzhu Meng, ${ }^{2}$ and Yulin Liu ${ }^{2}$ \\ ${ }^{1}$ College of Science, Shandong University of Science and Technology, Qingdao 266590, China \\ ${ }^{2}$ College of Information Science and Engineering, Shandong University of Science and Technology, Qingdao 266590, China \\ Correspondence should be addressed to Tongqian Zhang; zhangtongqian@sdust.edu.cn
}

Received 16 December 2012; Revised 18 June 2013; Accepted 23 June 2013

Academic Editor: Han H. Choi

Copyright (c) 2013 Wencai Zhao et al. This is an open access article distributed under the Creative Commons Attribution License, which permits unrestricted use, distribution, and reproduction in any medium, provided the original work is properly cited.

\begin{abstract}
SIR epidemic models with distributed delay are proposed. Firstly, the dynamical behaviors of the model without vaccination are studied. Using the Jacobian matrix, the stability of the equilibrium points of the system without vaccination is analyzed. The basic reproduction number $R$ is got. In order to study the important role of vaccination to prevent diseases, the model with distributed delay under impulsive vaccination is formulated. And the sufficient conditions of globally asymptotic stability of "infectionfree" periodic solution and the permanence of the model are obtained by using Floquet's theorem, small-amplitude perturbation skills, and comparison theorem. Lastly, numerical simulation is presented to illustrate our main conclusions that vaccination has significant effects on the dynamical behaviors of the model. The results can provide effective tactic basis for the practical infectious disease prevention.
\end{abstract}

\section{Introduction}

Infectious diseases have always been the problem that people have to face. Emerging diseases pose a continual threat to public health such as SARS and avian influenza. It is very necessary to establish and study mathematical models which can reflect the spread of the infectious diseases. A famous model in which the population is partitioned into three classes, the susceptible, infectious and recovered, with sizes denoted by $S, I$, and $R$, respectively, that could be used to describe an influenza epidemic was developed early in the 20th century by Kermack and McKendrick [1]. This model known as the susceptible-infectious-recovered (SIR) model is as follows:

$$
\begin{gathered}
S^{\prime}(t)=\Lambda-\beta S(t) I(t)-\mu S(t), \\
I^{\prime}(t)=\beta S(t) I(t)-r I(t)-\mu I(t), \\
R^{\prime}(t)=r I(t)-\mu R(t),
\end{gathered}
$$

where $S(t)$ denotes the number of members of a population susceptible to the disease, $I(t)$ denotes the total population of infectives with some virus at time $t, R(t)$ denotes the number of members who have been removed from the possibility of infection through a temporal immunity. In the model, parameters $\mu, \beta, r$, and $\Lambda$ are positive constants, where $\mu$ represents the death rates of susceptibles, infectives, and recovered. $\beta$ is the contact rate and $r$ is the recovery rate from the infected compartment. $\Lambda$ is the recruitment rate of the susceptible population. The SIR infectious disease model is a basic but important biologic model and has been studied by many authors [1-17]. Many diseases have the incubation period, such as rabies; the incubation period in rabies ranges from about two weeks to several months, and rarely even to years [18-21]. Diseases with incubation period always lead to time delay in the epidemic models, so delay differential equation is widely used in the epidemic mathematical model, such as $[2,7,8,10,12-16,22]$. Based on model (1), a new epidemic model with distributed delay is as follows:

$$
\begin{gathered}
S^{\prime}(t)=\Lambda-\beta I(t) \int_{-\infty}^{t} F(t-\tau) S(\tau) d \tau-\mu S(t), \\
I^{\prime}(t)=\beta I(t) \int_{-\infty}^{t} F(t-\tau) S(\tau) d \tau-r I(t)-\mu I(t), \\
R^{\prime}(t)=r I(t)-\mu R(t),
\end{gathered}
$$

where the function $F(t)=a e^{-a t}, a>0, \int_{0}^{+\infty} F(\tau) d \tau=1$. 
As is known to all, one of strategies to control infectious diseases is vaccination. Then a number of epidemic models in ecology can be formulated as dynamical systems of differential equations with vaccination [23-26]. Based on ODE, systems with sudden perturbations lead to impulsive differential equations. The theory of impulsive differential equations has been studied intensively and systematically in [27-34]. Compared to continuous constant vaccination, pulse vaccination seems more reasonable in the real world. Pulse vaccination, the repeated application of vaccine over a defined age range, is gaining prominence as a strategy for the elimination of childhood viral infections such as measles hepatitis, parotitis, smallpox, and phthisis. Under the pulse vaccination strategy (PVS) [9, 11, 17, 28, 35-41], what we are interested is how large a fraction of the population should we keep vaccinated in order to prevent the agent from establishing; that is, it is very important for us to investigate the conditions under which a given agent can invade a partially vaccinated population. Thus, we also need to consider the following epidemic model with distributed delay and pulse vaccination strategy at fixed moments, which is more realistic as

$$
\begin{gathered}
S^{\prime}(t)=\Lambda-\beta I(t) \int_{-\infty}^{t} F(t-\tau) S(\tau) d \tau-\mu S(t), \quad t \neq n T \\
I^{\prime}(t)=\beta I(t) \int_{-\infty}^{t} F(t-\tau) S(\tau) d \tau-r I(t)-\mu I(t), \quad t \neq n T \\
R^{\prime}(t)=r I(t)-\mu R(t), \quad t \neq n T, \\
S\left(t^{+}\right)=(1-\delta) S(t), \quad t=n T, \\
I\left(t^{+}\right)=I(t), \quad t=n T, \\
R\left(t^{+}\right)=R(t)+\delta S(t), \quad t=n T,
\end{gathered}
$$

where $\delta$ (with $0<\delta<1$ ) is the proportion of those vaccinated successfully to all of the susceptibles and $T$ is a fixed positive constant and denotes the period of the impulsive effect, $n=$ $\{1,2, \ldots\}$.

The organization of this paper is as follows. In Section 2, we will show the boundedness of the SIR system and give the transformation of models and some lemmas. In Section 3, we will analyze the local stability of equilibrium of system (7) and give the basic reproduction number. In Section 4.1, we will prove the existence and globally asymptotical stability of the periodic solution of the "infection-free" model. In Section 4.2, we obtain sufficient condition for the permanence of the epidemic model with pulse vaccination. Finally, we give numerical analysis and biological conclusions to show our main results.

\section{Transformation of Models and Prerequisites}

For system (2), the total population size $N(t)=S(t)+I(t)+$ $R(t)$ satisfies $N^{\prime}(t)=\Lambda-\mu N(t)$ and $\lim _{t \rightarrow \infty} N(t)=\Lambda / \mu$. Hence it is sufficient to consider system (2) with respect to
$D=\left\{(S, I, R) \in R_{+}^{3} \mid S(t)+I(t)+R(t) \leq(\Lambda / \mu)+\epsilon\right\}$. Note that the variable $R$ does not appear in the first and second equations of system (2); hence, we only need to consider the subsystem of (2) as follows:

$$
\begin{array}{r}
S^{\prime}(t)=\Lambda-\beta I(t) \int_{-\infty}^{t} F(t-\tau) S(\tau) d \tau-\mu S(t), \\
I^{\prime}(t)=\beta I(t) \int_{-\infty}^{t} F(t-\tau) S(\tau) d \tau-r I(t)-\mu I(t) .
\end{array}
$$

In order to study the system, we can use the chain transformation $Z(t)=\int_{-\infty}^{t} F(t-\tau) S(\tau) d \tau$. Since

$$
\int_{-\infty}^{t} F(t-\tau) d \tau=\lim _{A \rightarrow-\infty} \int_{A}^{t} a e^{-a(t-\tau)} d \tau=1
$$

and $\int_{-\infty}^{t} F(t-\tau) S(\tau) d \tau$ is convergent, then

$$
\Delta Z(t)=\int_{-\infty}^{t^{+}} F(t-\tau) S(\tau) d \tau-\int_{-\infty}^{t} F(t-\tau) S(\tau) d \tau=0
$$

Hence, system (4) becomes

$$
\begin{gathered}
S^{\prime}(t)=\Lambda-\beta I(t) Z(t)-\mu S(t), \\
I^{\prime}(t)=\beta I(t) Z(t)-r I(t)-\mu I(t), \\
Z^{\prime}(t)=a(S(t)-Z(t)) .
\end{gathered}
$$

We understand the relationship between the two systems as follows. If $(S, I):[0,+\infty) \rightarrow R^{2}$ is the solution of system (4) corresponding to continuous and bounded initial function $S(0)=S_{0}, I(0)=I_{0}$, then $(S, I, Z):[0,+\infty) \rightarrow R^{3}$ is a solution of system (7) with $S(0)=S_{0}, I(0)=I_{0}, Z(0)=Z_{0}$, and $Z(0)=\int_{-\infty}^{0} F(-\tau) S(\tau) d \tau=\int_{-\infty}^{0} a e^{a \tau} S(\tau) d \tau$. Conversely, if $(S, I, Z)$ is any solution of system (7) defined on the entire real line and bounded on $(-\infty, 0]$, then $Z$ is given by $Z(t)=$ $\int_{-\infty}^{t} F(t-\tau) S(\tau) d \tau$, and so $(S, I)$ satisfies system (4).

System (7) will be analyzed with the following initial conditions $S(0)=S_{0}>0, I(0)=I_{0}>0, Z(0)=Z_{0}>0$.

By the mean value theorem of integrals, there exists $\varsigma \in$ $(-\infty, t)$, such that

$$
Z(t)=\int_{-\infty}^{t} F(t-\tau) S(\tau) d \tau=S(\varsigma) \int_{-\infty}^{t} F(t-\tau) d \tau
$$

We know that $S(t)<(\Lambda / \mu)+\varepsilon$ and $\int_{-\infty}^{t} F(t-\tau) d \tau=1$; hence we get $Z(t)<(\Lambda / \mu)+\varepsilon$. 
By the same transformation, from system (3), we have

$$
\begin{gathered}
S^{\prime}(t)=\Lambda-\beta I(t) Z(t)-\mu S(t), \quad t \neq n T, \\
I^{\prime}(t)=\beta I(t) Z(t)-r I(t)-\mu I(t), \quad t \neq n T, \\
Z^{\prime}(t)=a(S(t)-Z(t)), \quad t \neq n T, \\
S\left(t^{+}\right)=(1-\delta) S(t), \quad t=n T, \\
I\left(t^{+}\right)=I(t), \quad t=n T, \\
Z\left(t^{+}\right)=Z(t), \quad t=n T .
\end{gathered}
$$

Motivated by the application of systems (9) to population dynamics (refer to [27]), we assume that solutions of systems (9) satisfy the initial conditions $\phi \in C_{h}^{+}$, and $\phi(0) \geq 0$ lie in $C_{h}^{+}=\left\{\phi=\left(\phi_{1}(s), \phi_{2}(s), \phi_{3}(s)\right) \in C_{h}: \phi_{i}(0) \geq 0(i=1,2,3)\right\}$, where $\phi_{i}(s)$ is positive, bounded, and continuous function for $s \in[0,+\infty)$.

We would like to have the following definitions first.

Definition 1. Let $R^{+}=[0, \infty)$. The map $V: R_{+} \times R_{+}^{3} \rightarrow R_{+}$is said to belong to class $V_{0}$ if

(i) $V$ is continuous in $(k T,(k+1) T] \times R_{+}^{3}, k \in N$, and for each $x \in R_{+}^{3}, \lim _{(t, z) \rightarrow\left(k T^{+}, x\right)} V(t, z)=V\left(k T^{+}, x\right)$ exist,

(ii) $V$ is locally Lipschitzian in $x$.

Also we have the following lemmas.

Lemma 2 (see [31]). Let $V: R_{+} \times R_{+}^{3} \rightarrow R_{+}$, and $V \in V_{0}$. Assume that

$$
\begin{aligned}
& D^{+} V(t, z(t)) \leq(\geq) g(t, V(t, z(t))), \quad t \neq n T, \\
& V\left(t, z\left(t^{+}\right)\right) \leq(\geq) \Psi_{n}(V(t, z(t))), \quad t=n T,
\end{aligned}
$$

where $g: R_{+} \times R_{+} \rightarrow R$ is continuous in $(n T,(n+1) T] \times R_{+}$ and for each $x \in R_{+}, n \in N, \lim _{(t, y) \rightarrow\left((n T)^{+}, x\right)} g(t, y)=$ $g\left((n T)^{+}, x\right)$ exist; $\Psi_{n}: R_{+} \rightarrow R_{+}$is nondecreasing. Let $r(t)=r\left(t, 0, u_{0}\right)$ be the maximal (minimal) solution of the scalar impulsive differential equation

$$
\begin{gathered}
u^{\prime}=g(t, u), \quad t \neq n T, \\
u\left(t^{+}\right)=\Psi_{n}(u(t)), \quad t=n T, \\
u\left(0^{+}\right)=u_{0},
\end{gathered}
$$

existing on $[0, \infty)$. Then $V\left(0^{+}, z_{0}\right) \leq(\geq) u_{0}$ implies that $V(t$, $z(t)) \leq(\geq) r(t), t \geq 0$, where $z(t)=z\left(t, 0, z_{0}\right)$ is any solution of $(9)$ existing on $[0, \infty)$.
Lemma 3. Consider the following system:

$$
\begin{gathered}
x^{\prime}(t)=\Lambda-\mu x(t), \quad t \neq n T, \\
y^{\prime}(t)=a(x(t)-y(t)), \quad t \neq n T, \\
x\left(t^{+}\right)=(1-\delta) x(t), \quad t=n T, \\
y\left(t^{+}\right)=y(t), \quad t=n T .
\end{gathered}
$$

Then system (12) has a unique positive T-periodic solution $x^{*}(t), y^{*}(t)$ as

$$
x^{*}(t)=\frac{\Lambda}{\mu}\left(1-\frac{\delta e^{-\mu(t-n T)}}{1-(1-\delta) e^{-\mu T}}\right),
$$

$y^{*}(t)$

$$
\begin{gathered}
=\frac{\Lambda}{\mu}\left(1+a \delta \frac{e^{-a(t-n T)}\left(1-e^{-\mu T}\right)-e^{-\mu(t-n T)}\left(1-e^{-a T}\right)}{(a-\mu)\left(1-e^{-a T}\right)\left(1-(1-\delta) e^{-\mu T}\right)}\right), \\
x^{*}\left(0^{+}\right)=\frac{\Lambda}{\mu}\left(1-\frac{\delta}{1-(1-\delta) e^{-\mu T}}\right), \\
y^{*}\left(0^{+}\right)=\frac{\Lambda}{\mu}\left(1-\frac{a \delta\left(e^{-\mu T}-e^{-a T}\right)}{(a-\mu)\left(1-e^{-a T}\right)\left(1-(1-\delta) e^{-\mu T}\right)}\right),
\end{gathered}
$$

and for each solution, $x(t) \rightarrow x^{*}(t), y(t) \rightarrow x^{*}(t)$ as $t \rightarrow$ $\infty$.

Proof. Solving the first equation of system (12), we have

$$
\begin{aligned}
x(t)= & x\left(n T^{+}\right) e^{-\mu(t-n T)} \\
& +\frac{\Lambda}{\mu}\left(1-e^{-\mu(t-n T)}\right), \quad n T<t \leq(n+1) T .
\end{aligned}
$$

Substituting $x(t)$ into the second equation of (12), we integrate both sides in interval $(n T,(n+1) T]$, and we get

$$
\begin{aligned}
y(t)= & y\left(n T^{+}\right) e^{-a(t-n T)} \\
& +\frac{a}{a-\mu}\left(x\left(n T^{+}\right)-\frac{\Lambda}{\mu}\right)\left(e^{-\mu(t-n T)}-e^{-a(t-n T)}\right) \\
& +\frac{\Lambda}{\mu}\left(1-e^{-a(t-n T)}\right) .
\end{aligned}
$$


By using stroboscopic map of difference equation, we have

$$
\begin{aligned}
x\left((n+1) T^{+}\right)= & (1-\delta) x((n+1) T) \\
= & (1-\delta) x\left(n T^{+}\right) e^{-\mu T} \\
& +\frac{\Lambda}{\mu}(1-\delta)\left(1-e^{-\mu T}\right), \\
y\left((n+1) T^{+}\right)= & y((n+1) T) \\
= & y\left(n T^{+}\right) e^{-a T}+\frac{a}{a-\mu}\left(x\left(n T^{+}\right)-\frac{\Lambda}{\mu}\right) \\
& \times\left(e^{-\mu T}-e^{-a T}\right)+\frac{\Lambda}{\mu}\left(1-e^{-a T}\right) .
\end{aligned}
$$

The fixed point of the above mapping is

$$
\begin{gathered}
x^{*}=\frac{\Lambda}{\mu}\left(1-\frac{\delta}{1-(1-\delta) e^{-\mu T}}\right), \\
y^{*}=\frac{\Lambda}{\mu}\left(1-\frac{a \delta\left(e^{-\mu T}-e^{-a T}\right)}{(a-\mu)\left(1-e^{-a T}\right)\left(1-(1-\delta) e^{-\mu T}\right)}\right) .
\end{gathered}
$$

Therefore, we can get the following $T$-periodic solution $\left(x^{*}(t), y^{*}(t)\right)$ of system (12):

$$
\begin{array}{r}
x^{*}(t)=\frac{\Lambda}{\mu}\left(1-\frac{\delta e^{-\mu(t-n T)}}{1-(1-\delta) e^{-\mu T}}\right), \quad n T<t \leq(n+1) T, \\
y^{*}(t) \\
=\frac{\Lambda}{\mu}\left(1+a \delta \frac{\left(1-e^{-\mu T}\right) e^{-a(t-n T)}-\left(1-e^{-a T}\right) e^{-\mu(t-n T)}}{(a-\mu)\left(1-e^{-a T}\right)\left(1-(1-\delta) e^{-\mu T}\right)}\right), \\
n T<t \leq(n+1) T .
\end{array}
$$

Next, we will prove the attractivity of periodic solutions. Let $(x(t), y(t))$ be an any solution of the system (12); then, for $t \in$ $(n T,(n+1) T]$, we have

$$
\begin{gathered}
x(t)=x^{*}(t)+\left(x\left(n T^{+}\right)-x^{*}\right) e^{-\mu(t-n T)}, \\
y(t)=y^{*}(t)+\left(y\left(n T^{+}\right)-y^{*}\right) e^{-a(t-n T)} \\
+\frac{a}{a-\mu}\left(x\left(n T^{+}\right)-x^{*}\right)\left(e^{-\mu(t-n T)}-e^{-a(t-n T)}\right) .
\end{gathered}
$$

On the one hand, by the recurrence formula, we have

$$
x\left((n+1) T^{+}\right)=(1-\delta) e^{-\mu T} x\left(n T^{+}\right)+\frac{\Lambda}{\mu}(1-\delta)\left(1-e^{-\mu T}\right) .
$$

Thus

$$
\begin{aligned}
x\left(n T^{+}\right)= & (1-\delta)^{n} e^{-n \mu T} x\left(0^{+}\right) \\
& +\left[1+(1-\delta) e^{-\mu T}+\cdots+(1-\delta)^{n-1} e^{-(n-1) \mu T}\right] \\
& \times \frac{\Lambda}{\mu}(1-\delta)\left(1-e^{-\mu T}\right) \\
= & (1-\delta)^{n} e^{-n \mu T} x\left(0^{+}\right) \\
& +\frac{\Lambda}{\mu}(1-\delta)\left(1-e^{-\mu T}\right) \frac{1-(1-\delta)^{n} e^{-n \mu T}}{1-(1-\delta) e^{-\mu T}} \\
\longrightarrow & x^{*} \quad(n \longrightarrow \infty),
\end{aligned}
$$

and then we have $x(t) \rightarrow x^{*}(t)(n \rightarrow \infty)$.

On the other hand,

$$
\begin{aligned}
y\left((n+1) T^{+}\right)= & y((n+1) T) \\
= & y\left(n T^{+}\right) e^{-a T}+\frac{a}{a-\mu}\left(x\left(n T^{+}\right)-\frac{\Lambda}{\mu}\right) \\
& \times\left(e^{-\mu T}-e^{-a T}\right)+\frac{\Lambda}{\mu}\left(1-e^{-a T}\right),
\end{aligned}
$$

and $\lim _{n \rightarrow \infty} x\left(n T^{+}\right)=x^{*}$, for $n$ large enough we have the following approximate recursive formula:

$$
\begin{aligned}
y\left((n+1) T^{+}\right)= & y\left(n T^{+}\right) e^{-a T}+\frac{a}{a-\mu}\left(x^{*}-\frac{\Lambda}{\mu}\right) \\
& \times\left(e^{-\mu T}-e^{-a T}\right)+\frac{\Lambda}{\mu}\left(1-e^{-a T}\right) \\
= & y\left(n T^{+}\right) e^{-a T}+\frac{a \delta \Lambda\left(e^{-a T}-e^{-\mu T}\right)}{\mu(a-\mu)\left(1-(1-\delta) e^{-\mu T}\right)} \\
& +\frac{\Lambda}{\mu}\left(1-e^{-a T}\right) .
\end{aligned}
$$

Thus, for any $m \in N^{+}$, we get

$$
\begin{aligned}
y\left((m+N) T^{+}\right) \\
=y\left(N T^{+}\right) e^{-m a T} \\
+\left[1+e^{-a T}+\cdots+e^{-(m-1) a T}\right] \\
\quad \times\left(\frac{a \delta \Lambda\left(e^{-a T}-e^{-\mu T}\right)}{\mu(a-\mu)\left(1-(1-\delta) e^{-\mu T}\right)}+\frac{\Lambda}{\mu}\left(1-e^{-a T}\right)\right)
\end{aligned}
$$




$$
\begin{aligned}
= & y\left(N T^{+}\right) e^{-m a T} \\
& +\frac{1-e^{-m a T}}{1-e^{-a T}} \\
& \times\left(\frac{a \delta \Lambda\left(e^{-a T}-e^{-\mu T}\right)}{\mu(a-\mu)\left(1-(1-\delta) e^{-\mu T}\right)}+\frac{\Lambda}{\mu}\left(1-e^{-a T}\right)\right) \\
\longrightarrow & \frac{\Lambda}{\mu}\left(1-\frac{a \delta\left(e^{-\mu T}-e^{-a T}\right)}{(a-\mu)\left(1-e^{-a T}\right)\left(1-(1-\delta) e^{-\mu T}\right)}\right) \\
& \quad(m \longrightarrow \infty) .
\end{aligned}
$$

Then we have $\lim _{n \rightarrow \infty} y\left(n T^{+}\right)=y^{*}$, thus $y(t) \rightarrow y^{*}(t)$ $(t \rightarrow \infty)$. The proof is completed.

\section{The Stability of Equilibrium of System (7) and the Basic Reproduction Number}

In this section, we will consider the local stability of equilibrium of system (7) and give the basic reproduction number. Obviously, the system (7) has a disease-free equilibrium $E_{0}(\Lambda / \mu, 0, \Lambda / \mu)$ and an endemic equilibrium $E^{*}\left(S^{*}, I^{*}, Z^{*}\right)$, where $S^{*}=(\mu+r) / \beta, I^{*}=(\beta \Lambda-\mu(\mu+r)) / \beta(\mu+r), Z^{*}=$ $(\mu+r) / \beta$. Let $R=\beta \Lambda / \mu(\mu+r)$; we have the following theorem.

Theorem 4. System (7) always has a disease-free equilibrium $E_{0}$. If and only if $R>1$, system (7) has an endemic equilibrium $E^{*}$.

3.1. Local Stability of Disease-Free Equilibrium $E_{0}$. We calculate the Jacobian matrix of system (7) evaluated at $E_{0}$; one gets the following matrix:

$$
J\left(E_{0}\right)=\left(\begin{array}{ccc}
-\mu & -\beta \frac{\Lambda}{\mu} & 0 \\
0 & \beta \frac{\Lambda}{\mu}-\mu-r & 0 \\
a & 0 & -a
\end{array}\right) .
$$

Obviously, $E_{0}$ is locally asymptotically stable if $\beta(\Lambda / \mu)-\mu-$ $r<0$ which implies that $R<1$, and unstable if $R>1$. Then $R=\beta \Lambda / \mu(\mu+r)$ can be used as the basic reproductive number. Thus, we obtain the following result.

Theorem 5. If $R<1$, then disease-free equilibrium $E_{0}$ of system (7) is locally asymptotically stable and unstable if $R>1$.

3.2. Local Stability of Endemic Equilibrium $E^{*}$. About the local stability of endemic equilibrium $E^{*}$, we have the following theorem.

Theorem 6. If $R>1$ and $a \geq r$ or $1<R<(r+\mu) /(r-a)$, the equilibrium $E^{*}$ of system (7) is locally asymptotically stable.
Proof. The Jacobian matrix of system (7) evaluated at $E^{*}$ is

$$
J\left(E^{*}\right)=\left(\begin{array}{ccc}
-\mu & -\beta Z^{*} & -\beta I^{*} \\
0 & 0 & \beta I^{*} \\
a & 0 & -a
\end{array}\right) .
$$

Let $\lambda_{i}(i=1,2,3)$ be its eigenvalues with $\operatorname{Re} \lambda_{1} \leq \operatorname{Re} \lambda_{2} \leq$ $\operatorname{Re} \lambda_{3}$. After a simple calculation, it follows that

$$
\operatorname{det} J\left(E^{*}\right)=-a \beta^{2} I^{*} Z^{*}<0 .
$$

For $\operatorname{det} J\left(E^{*}\right)=\lambda_{1} \lambda_{2} \lambda_{3}<0$, there are two cases as follows:

(i) $\operatorname{Re} \lambda_{i}<0$ for $i=1,2,3$;

(ii) $\operatorname{Re} \lambda_{1}<0 \leq \operatorname{Re} \lambda_{2} \leq \operatorname{Re} \lambda_{3}$.

Now we prove that the case (ii) is not true. Note that $\operatorname{det} J\left(E^{*}\right)=-a \beta^{2} I^{*} Z^{*}<0$; one gets $\operatorname{tr} J\left(E^{*}\right)<0$, that is, $\lambda_{1}+\lambda_{2}+\lambda_{3}<0$. If the case (ii) is true, we have that $\operatorname{Re}\left(\lambda_{1}+\right.$ $\left.\lambda_{2}\right)<0$ and $\operatorname{Re}\left(\lambda_{1}+\lambda_{3}\right)<0$. The second additive compound matrix [6] of $J\left(E^{*}\right)$ (see the Appendix) is as follows:

$$
\begin{aligned}
J^{[2]}\left(E^{*}\right) & =\left(\begin{array}{ccc}
-\mu & \beta I^{*} & \beta I^{*} \\
0 & -\mu-a & -\beta Z^{*} \\
-a & 0 & -a
\end{array}\right), \\
\operatorname{det} J^{[2]}\left(E^{*}\right)= & -a\left(\left(\beta I^{*}+\mu\right)(\mu+a)-\beta^{2} I^{*} Z^{*}\right) \\
= & -a \mu(R(a-r)+(\mu+r)),
\end{aligned}
$$

where $S^{*}=(\mu+r) / \beta, I^{*}=(\beta \Lambda-\mu(\mu+r)) / \beta(\mu+r)$, and $Z^{*}=(\mu+r) / \beta$ is used. Notice that $R>1$, and two cases will happen as follows:

(a) if $a \geq r$, then $\operatorname{det} J^{[2]}\left(E^{*}\right)<0$;

(b) if $a<r$, but $1<R<(r+\mu) /(r-a)$, then $\operatorname{det} J^{[2]}\left(E^{*}\right)<$ 0 .

According to the property of the second additive compound matrix [6], the eigenvalues of $J^{[2]}\left(E^{*}\right)$ are $\lambda_{i}+\lambda_{j}, 1 \leq i<j \leq$ 3. Then, we have

$$
\left(\lambda_{1}+\lambda_{2}\right)\left(\lambda_{1}+\lambda_{3}\right)\left(\lambda_{2}+\lambda_{3}\right)<0 .
$$

Notice that $\operatorname{Re}\left(\lambda_{1}+\lambda_{2}\right)<0$ and $\operatorname{Re}\left(\lambda_{1}+\lambda_{3}\right)<0$, then we get $\operatorname{Re}\left(\lambda_{2}+\lambda_{3}\right)<0$, which contradicts with case (ii). Therefore, $\operatorname{Re} \lambda_{i}<0$ for $i=1,2,3$. So $E^{*}$ is locally asymptotically stable for $R>1$ and $a \geq r$ or $1<R<(r+\mu) /(r-a)$. This completes the proof.

3.3. Analysis at $R=1$. In this section, we consider the stability of system (7) under $R=1$ using the center manifold theory, as described in [42, Theorem 4.1] . To apply this method, the following simplification and change of variables are made first. Let $S=x_{1}, I=x_{2}, Z=x_{3}$, and the system (7) becomes

$$
\begin{gathered}
\dot{x}_{1}=\Lambda-\beta x_{2} x_{3}-\mu x_{1}=f_{1}, \\
\dot{x}_{2}=\beta x_{2} x_{3}-r x_{2}-\mu x_{2}=f_{2}, \\
\dot{x}_{3}=a\left(x_{1}-x_{3}\right)=f_{3},
\end{gathered}
$$


with $R=1$ corresponding to $\beta=\beta^{*}=\mu(\mu+r) / \Lambda$. The virusfree equilibrium is $E_{0}(\Lambda / \mu, 0, \Lambda / \mu)$. The linearization matrix of system (7) around the infection-free equilibrium $E_{0}$ when $\beta=\beta^{*}$ is

$$
\begin{aligned}
J(f) & =\left(\begin{array}{ccc}
-\mu & -\frac{\beta^{*} \Lambda}{\mu} & 0 \\
0 & \frac{\beta^{*} \Lambda}{\mu}-(\mu+r) & 0 \\
a & 0 & -a
\end{array}\right) \\
& =\left(\begin{array}{ccc}
-\mu & -(\mu+r) & 0 \\
0 & 0 & 0 \\
a & 0 & -a
\end{array}\right) .
\end{aligned}
$$

The matrix $J(f)$ has eigenvalues $(0,-\mu,-a)^{T}$, which meets the requirement of a simple zero eigenvalue and others having negative real part. A right eigenvector $\eta$ corresponding to the zero eigenvalue is $\eta=(-(\mu+r) / \mu, 1,-(\mu+r) / \mu)^{T}$ and the left eigenvector satisfying $\xi \eta=1$ is $\xi=(0,1,0)$. For the system (7), we can get

$$
\begin{gathered}
a=\sum_{i, j, k=1}^{3} \xi_{k} \eta_{i} \eta_{j} \frac{\partial^{2} f_{k}}{\partial x_{i} \partial x_{j}}\left(\frac{\Lambda}{\mu}, 0, \frac{\Lambda}{\mu}\right)=-\frac{2}{\Lambda}(\mu+r)^{2}<0, \\
b=\sum_{i, k=1}^{3} \xi_{k} \eta_{i} \frac{\partial^{2} f_{k}}{\partial x_{i} \partial \beta}\left(\frac{\Lambda}{\mu}, 0, \frac{\Lambda}{\mu}\right)=\frac{\Lambda}{\mu}>0 .
\end{gathered}
$$

Thus, $a<0, b>0$, by item (iv) of Theorem 4.1 in [42]; we can give the following result.

Theorem 7. The disease-free equilibrium $E_{0}$ for system (7) is locally asymptotically stable for $R$ near 1 .

\section{Disease Impulsive Control for System (9)}

4.1. The Existence and Globally Asymptotical Stability of the "Infection-Free" Periodic Solution of System (9). We demonstrate the expression of the infectives-free solution of the system (9) firstly, in which the infectives are entirely absent from the population permanently. Consider the infectivesfree subsystem of system (9) in the form

$$
\begin{gathered}
S^{\prime}(t)=\Lambda-\mu S(t), \quad t \neq n T, \\
Z^{\prime}(t)=a(S(t)-Z(t)), \quad t \neq n T, \\
S\left(t^{+}\right)=(1-\delta) S(t), \quad t=n T, \\
Z\left(t^{+}\right)=Z(t), \quad t=n T .
\end{gathered}
$$

By Lemma 3, system (33) has a unique positive $T$-periodic solution $\left(S^{*}(t), Z^{*}(t)\right)$ given by

$$
\begin{aligned}
& S^{*}(t)=\frac{\Lambda}{\mu}\left(1-\frac{\delta e^{-\mu(t-n T)}}{1-(1-\delta) e^{-\mu T}}\right), \quad n T<t \leq(n+1) T, \\
& Z^{*}(t) \\
& =\frac{\Lambda}{\mu}\left(1+a \delta \frac{e^{-a(t-n T)}\left(1-e^{-\mu T}\right)-e^{-\mu(t-n T)}\left(1-e^{-a T}\right)}{(a-\mu)\left(1-e^{-a T}\right)\left(1-(1-\delta) e^{-\mu T}\right)}\right), \\
& S^{*}\left(0^{+}\right)=\frac{\Lambda}{\mu}\left(1-\frac{\delta}{1-(1-\delta) e^{-\mu T}}\right), \\
& Z^{*}\left(0^{+}\right)=\frac{\Lambda}{\mu}\left(1-\frac{a \delta\left(e^{-\mu T}-e^{-a T}\right)}{(a-\mu)\left(1-e^{-a T}\right)\left(1-(1-\delta) e^{-\mu T}\right)}\right),
\end{aligned}
$$

and for each solution, $S(t) \rightarrow S^{*}(t), Z(t) \rightarrow Z^{*}(t)$ as $t \rightarrow$ $\infty$. Hence, we have Theorem 8 .

Theorem 8. The system (9) has an "infection-free" periodic solution $\left(S^{*}(t), 0, Z^{*}(t)\right)$ for $t \in(n T,(n+1) T], n \in N$.

In next section, we will prove that "infection-free" periodic solution $\left(S^{*}(t), 0, Z^{*}(t)\right)$ is globally asymptotically stable.

Theorem 9. Let $(S(t), I(t), Z(t))$ be any solution of (9), and then $\left(S^{*}(t), 0, Z^{*}(t)\right)$ is globally asymptotically stable provided $\Re<1$, where $\mathfrak{R}=\left((\beta \Lambda / \mu)\left(T-\delta\left(1-e^{-\mu T}\right) / \mu(1-(1-\right.\right.$ $\left.\left.\left.\delta) e^{-\mu T}\right)\right)\right) /(\mu+r) T$.

Proof. Firstly, we will prove the local stability. The local stability of periodic solution $\left(S^{*}(t), 0, Z^{*}(t)\right)$ may be determined by considering the behavior of small amplitude perturbations of the solution. This may be written as

$$
\left(\begin{array}{c}
u(t) \\
v(t) \\
w(t)
\end{array}\right)=\Phi(t)\left(\begin{array}{c}
u(0) \\
v(0) \\
w(0)
\end{array}\right), \quad 0 \leq t<T
$$

where $\Phi$ satisfies

$$
\frac{d \Phi(t)}{d t}=\left(\begin{array}{ccc}
-\mu & -\beta Z^{*}(t) & 0 \\
0 & \beta Z^{*}(t)-\mu-r & 0 \\
a & 0 & -a
\end{array}\right) \Phi(t)
$$

and $\Phi(0)=E$, the identity matrix. Hence, the fundamental solution matrix is

$$
\Phi(t)=\left(\begin{array}{ccc}
e^{-\mu t} & A & 0 \\
0 & e^{\int_{0}^{t}\left(\beta Z^{*}(s)-\mu-r\right) d s} & 0 \\
C & 0 & e^{-a t}
\end{array}\right) .
$$


There is no need to calculate the exact form of $A, C$ as it is not required in the analysis that follows. The linearization of the fourth, fifth, and sixth equations of system (9) becomes

$$
\left(\begin{array}{c}
u\left(n T^{+}\right) \\
v\left(n T^{+}\right) \\
w\left(n T^{+}\right)
\end{array}\right)=\left(\begin{array}{ccc}
1-\delta & 0 & 0 \\
0 & 1 & 0 \\
0 & 0 & 1
\end{array}\right)\left(\begin{array}{c}
u(n T) \\
v(n T) \\
w(n T)
\end{array}\right) .
$$

The stability of the periodic solution $\left(S^{*}(t), 0, Z^{*}(t)\right)$ is determined by the eigenvalues of

$$
M=\left(\begin{array}{ccc}
1-\delta & 0 & 0 \\
0 & 1 & 0 \\
0 & 0 & 1
\end{array}\right) \Phi(T),
$$

which are

$$
\begin{gathered}
\lambda_{1}=(1-\delta) e^{-\mu T}<1, \quad \lambda_{2}=e^{-a T}<1, \\
\lambda_{3}=e^{\int_{0}^{T}\left(\beta Z^{*}(t)-\mu-r\right) d t} .
\end{gathered}
$$

According to Floquet theory (see [43]), $\left(S^{*}(t), 0, Z^{*}(t)\right)$ is locally stable if $\left|\lambda_{3}\right|<1$.

Denote

$$
\mathfrak{R}=\frac{(\beta \Lambda / \mu)\left(T-\delta\left(1-e^{-\mu T}\right) / \mu\left(1-(1-\delta) e^{-\mu T}\right)\right)}{(\mu+r) T},
$$

For $\mathfrak{R}<1$, we have

$$
\int_{0}^{T}\left(\beta Z^{*}(t)-\mu-r\right) d t<0
$$

which leads to $\left|\lambda_{3}\right|<1$. Thus the periodic solution $\left(S^{*}(t), 0\right.$, $\left.Z^{*}(t)\right)$ is locally stable.

In the following, we prove the global attractivity. Since $\mathfrak{R}<1$ holds, we have

$$
\frac{\beta \Lambda}{\mu}\left(T-\frac{\delta\left(1-e^{-\mu T}\right)}{\mu\left(1-(1-\delta) e^{-\mu T}\right)}\right)-(\mu+r) T<0 .
$$

We can choose a $\varepsilon>0$ small enough such that

$$
\begin{aligned}
\eta= & \beta \frac{\Lambda}{\mu}\left((1+2 \varepsilon) T-\frac{\delta\left(1-e^{-\mu T}\right)}{\mu\left(1-(1-\delta) e^{-\mu T}\right)}\right) \\
& -(\mu+r) T<0 .
\end{aligned}
$$

Note that $S^{\prime}(t) \leq \Lambda-\mu S(t)$, by impulsive differential inequalities, we have

$$
S(t)<S^{*}(t)+\varepsilon
$$

for all $t$ large enough. For simplification, we may assume that (45) holds for all $t \geq 0$. From the third equation of system (9) and (45), we get

$$
\begin{gathered}
Z^{\prime}(t) \leq a\left(S^{*}(t)+\varepsilon-Z(t)\right), \quad t \neq n T, \\
Z\left(t^{+}\right)=Z(t), \quad t=n T .
\end{gathered}
$$

By impulsive differential inequalities, we have

$$
Z(t)<P^{*}(t)+\varepsilon,
$$

where $P^{*}(t)$ is the periodic solution of the following equation:

$$
\begin{gathered}
P^{\prime}(t)=a\left(S^{*}(t)+\varepsilon-P(t)\right), \quad t \neq n T, \\
P\left(t^{+}\right)=P(t), \quad t=n T,
\end{gathered}
$$

$P^{*}(t)$

$$
\begin{aligned}
= & \frac{\Lambda}{\mu}\left(1+a \delta \frac{e^{-a(t-n T)}\left(1-e^{-\mu T}\right)-e^{-\mu(t-n T)}\left(1-e^{-a T}\right)}{(a-\mu)\left(1-e^{-a T}\right)\left(1-(1-\delta) e^{-\mu T}\right)}\right) \\
& +\varepsilon, \quad n T<t \leq(n+1) T .
\end{aligned}
$$

From the second and fifth equations of the system (9), we have

$$
\begin{gathered}
I^{\prime}(t) \leq \beta I(t)\left(P^{*}(t)+\varepsilon\right)-\mu I(t)-r I(t), \quad t \neq n T, \\
I\left(t^{+}\right)=I(t), \quad t=n T,
\end{gathered}
$$

which leads to

$I((n+1) T)$

$$
\begin{aligned}
& \leq I\left(n T^{+}\right) \exp \int_{n T}^{(n+1) T}\left(\beta\left(P^{*}(t)+\varepsilon\right)-(\mu+r)\right) d t \\
& =I(n T) \exp \int_{n T}^{(n+1) T}\left(\beta\left(P^{*}(t)+\varepsilon\right)-(\mu+r)\right) d t \\
& =I(n T) e^{\eta} .
\end{aligned}
$$

Hence $I(n T) \leq I(0) e^{n \eta}$ and $I(n T) \rightarrow 0$ as $n \rightarrow+\infty$. Therefore, $I(t) \rightarrow 0$ as $t \rightarrow+\infty$, since $I(t) \leq I(n T)$ for $n T<t \leq(n+1) T$.

Next, we prove that $S(t) \rightarrow S^{*}(t), Z(t) \rightarrow Z^{*}(t)$ as $t \rightarrow$ $+\infty$. For all $\varepsilon>0$, there must exist a $T_{1}>0$ such that $0 \leq$ $I(t)<\varepsilon$ for $t>T_{1}$. Without loss of generality, we may assume that $0 \leq I(t)<\varepsilon$ for all $t \geq 0$, and then from system (9), we have

$$
\Lambda-\frac{\beta \Lambda \varepsilon}{\mu}-\mu S(t) \leq S^{\prime}(t) \leq \Lambda-\mu S(t) .
$$

Then we have $q(t)<S(t)<S^{*}(t)+\varepsilon_{1}$ and $q(t) \rightarrow q^{*}(t)$ as $t \rightarrow+\infty$, where $q(t)$ is the solution of

$$
\begin{gathered}
q^{\prime}(t)=\Lambda-\frac{\beta \Lambda \varepsilon}{\mu}-\mu q(t), \quad t \neq n T, \\
q\left(t^{+}\right)=(1-\delta) q(t), \quad t=n T, \\
q\left(0^{+}\right)=S\left(0^{+}\right), \\
q^{*}(t)=\frac{\Lambda(\mu-\beta \varepsilon)}{\mu^{2}}\left(1-\frac{\delta e^{-\mu(t-n T)}}{1-(1-\delta) e^{-\mu T}}\right), \\
n T<t \leq(n+1) T .
\end{gathered}
$$


Therefore, for any $\varepsilon_{1}>0$, there exists a $T_{2}>0$ such that $q^{*}(t)-\varepsilon_{1}<S(t)<S^{*}(t)+\varepsilon_{1}$. Let $\varepsilon \rightarrow 0$; we have $S^{*}(t)-\varepsilon_{1} \leq$ $S(t) \leq S^{*}(t)+\varepsilon_{1}$ for $t$ large enough, which implies $S(t) \rightarrow$ $S^{*}(t)$ as $t \rightarrow+\infty$.

Similarly, $Z(t) \rightarrow Z^{*}(t)$ as $t \rightarrow+\infty$ can be analyzed by the same method as the above, so we omit it. This completes the proof.

\subsection{Permanence of System (9)}

Theorem 10. If $\Re>1$, then system (9) is permanent, that is, there exist three positive constants $m_{1}, m_{2}$, and $m_{3}$ such that $S(t) \geq m_{1}, I(t) \geq m_{2}$, and $Z(t) \geq m_{3}$ for t large enough.

Proof. Suppose that the $X(t)=(S(t), I(t), Z(t))$ is any positive solution of system (9). From system (9), we can get

$$
\begin{gathered}
S^{\prime}(t) \geq \Lambda-\frac{\beta \Lambda^{2}}{\mu^{2}}-\mu S(t), \quad t \neq n T, \\
S\left(t^{+}\right)=(1-\delta) S(t), \quad t=n T .
\end{gathered}
$$

Consider the following impulsive differential equation:

$$
\begin{gathered}
G^{\prime}(t)=\Lambda-\frac{\beta \Lambda^{2}}{\mu^{2}}-\mu G(t), \quad t \neq n T, \\
G\left(t^{+}\right)=(1-\delta) G(t), \quad t=n T, \\
G\left(0^{+}\right)=S\left(0^{+}\right) .
\end{gathered}
$$

Then, we have $S(t) \geq G(t)>G^{*}(t)-\varepsilon$, where

$$
\begin{gathered}
G^{*}(t)=\frac{\Lambda-\beta \Lambda^{2} / \mu^{2}}{\mu}\left(1-\frac{\delta e^{-\mu(t-n T)}}{1-(1-\delta) e^{-\mu T}}\right), \\
n T<t \leq(n+1) T, \\
G_{0}^{*}=\frac{\left(\Lambda-\beta \Lambda^{2} / \mu^{2}\right)(1-\delta)\left(1-e^{-\mu T}\right)}{\mu\left(1-(1-\delta) e^{-\mu T}\right)} .
\end{gathered}
$$

So we have

$$
S(t) \geq G(t)>G^{*}(t)-\varepsilon>G_{0}^{*}-\varepsilon=m_{1}>0
$$

for $t$ large enough. From (56) and the third and sixth equations of the system (9), we have

$$
\begin{gathered}
Z^{\prime}(t)>a\left(m_{1}-Z(t)\right), \quad t \neq n T, \\
Z\left(t^{+}\right)=Z(t), \quad t=n T .
\end{gathered}
$$

Consider the following system:

$$
\begin{gathered}
H^{\prime}(t)=a\left(m_{1}-H(t)\right), \quad t \neq n T, \\
H\left(t^{+}\right)=Z(t), \quad t=n T .
\end{gathered}
$$

Obviously, $H(t) \rightarrow m_{1}$ as $t \rightarrow \infty$, Then there exists $\varepsilon>0$ such that

$$
Z(t)>m_{1}-\varepsilon=m_{3}>0 .
$$

Next, we prove that there exists a constant $m_{2}>0$ such that $I(t) \geq m_{2}$ for $t$ large enough. We will do it in the following two steps.

Step (I). Since $\mathfrak{R}>1$, we can choose $m_{4}, \varepsilon>0$ small enough such that

$$
\begin{aligned}
\omega= & \beta\left(\left(\frac{\Lambda-\beta m_{4}}{\mu}-2 \varepsilon\right) T-\frac{\delta\left(\Lambda-\beta m_{4}\right)\left(1-e^{-\mu T}\right)}{\mu^{2}\left(1-(1-\delta) e^{-\mu T}\right)}\right) \\
& -(\mu+r) T>0 .
\end{aligned}
$$

We will prove that $I(t)<(\mu / \Lambda) m_{4}$ cannot hold for all $t \geq 0$. Otherwise,

$$
\begin{gathered}
S^{\prime}(t) \geq \Lambda-\beta m_{4}-\mu S(t), \quad t \neq n T, \\
S\left(t^{+}\right)=(1-\delta) S(t), \quad t=n T .
\end{gathered}
$$

So we have $S(t) \geq m(t)>m^{*}(t)-\varepsilon$ for $t$ large enough, where $m(t)$ is the solution of

$$
\begin{gathered}
m^{\prime}(t)=\Lambda-\beta m_{4}-\mu m(t), \quad t \neq n T, \\
m\left(t^{+}\right)=(1-\delta) m(t), \quad t=n T, \\
m^{*}(t)=\frac{\Lambda-\beta m_{4}}{\mu}\left(1-\frac{\delta e^{-\mu(t-n T)}}{1-(1-\delta) e^{-\mu T}}\right), \\
m^{*}=\frac{n T<t \leq(n+1) T,}{\mu}\left(1-\frac{\delta}{1-(1-\delta) m^{-\mu T}}\right) .
\end{gathered}
$$

From the third and sixth equations of the system (9), we have

$$
\begin{gathered}
Z^{\prime}(t) \geq a\left(m^{*}(t)-\varepsilon-Z(t)\right), \quad t \neq n T, \\
Z\left(t^{+}\right)=Z(t), \quad t=n T,
\end{gathered}
$$

and $Z(t)>h^{*}(t)-\varepsilon$, where

$$
\begin{aligned}
h^{*}(t)= & \frac{\Lambda-\beta m_{4}}{\mu}+\frac{\Lambda-\beta m_{4}}{\mu} a \delta \\
& \times\left(\frac{\left(1-e^{-\mu T}\right) e^{-a(t-n T)}-\left(1-e^{-a T}\right) e^{-\mu(t-n T)}}{(a-\mu)\left(1-e^{-a T}\right)\left(1-(1-\delta) e^{-\mu T}\right)}\right)-\varepsilon .
\end{aligned}
$$

So we have

$$
\begin{gathered}
I^{\prime}(t) \geq\left(\beta\left(h^{*}(t)-\varepsilon\right)-\mu-r\right) I(t), \quad t \neq n T, \\
I\left(t^{+}\right)=I(t), \quad t=n T .
\end{gathered}
$$

Integrating (66) on $(n T,(n+1) T]$, we have

$$
\begin{aligned}
& I(n+1) T \\
& \quad \geq I\left(n T^{+}\right) \exp \int_{n T}^{(n+1) T}\left(\beta\left(h^{*}(t)-\varepsilon\right)-\mu-r\right) d t \\
& \quad=I(n T) e^{\omega} .
\end{aligned}
$$


Then $I(n T) \geq I(0) e^{n \omega} \rightarrow \infty$ as $n \rightarrow \infty$, which is a contradiction to the boundedness of $I(t)$. Hence, there exists a $t_{1}>0$ such that $I\left(t_{1}\right) \geq(\mu / \Lambda) m_{4}$. For the sake of simplification, we let $(\mu / \Lambda) m_{4}$ be $m_{4}$.

Step (II). If $I(t) \geq m_{4}$ for all $t \geq t_{1}$, and we let $m_{2}=m_{4}$, then our aim is obtained. Otherwise, let $t^{*}=\inf _{t>t_{1}}\{t \mid I(t)<$ $\left.m_{4}\right\}$, there are two possible case for $t^{*}$.

Case (I). $t^{*}=n_{1} T, n_{1} \in Z^{+}$. Then $I(t) \geq m_{4}$ for $t \in\left[t_{1}, t^{*}\right)$ and $I\left(t^{*}\right)=m_{4}$. Choose $n_{2}, n_{3} \in Z_{+}$, such that

$$
n_{2} T>\frac{1}{\mu} \ln \frac{1+m^{*}}{\varepsilon}, \quad e^{-\left(n_{2}+1\right)(\mu+r) T} e^{n_{3} \omega}>1 .
$$

Let $T^{\prime}=\left(n_{2}+n_{3}\right) T$, we claim that there exists a $t_{2} \epsilon$ $\left(t^{*}, t^{*}+T^{\prime}\right]$ such that $I\left(t_{2}\right)>m_{4}$. Otherwise, consider (62) with $m\left(n_{1} T^{+}\right)=S\left(n_{1} T^{+}\right)$for $t \in(n T,(n+1) T]$ and $n_{1} \leq n \leq$ $n_{1}+n_{2}+n_{3}$. We have

$$
\left|m(t)-m^{*}(t)\right| \leq\left(1+m^{*}\right) e^{-\mu\left(t-t^{*}\right)}<\varepsilon
$$

for $\left(n_{1}+n_{2}\right) T<t \leq\left(n_{1}+n_{2}+n_{3}\right) T$. So as in the above step (I), we have

$$
I\left(t^{*}+T^{\prime}\right) \geq I\left(t^{*}+n_{2} T\right) e^{n_{3} \omega} .
$$

From system (9), we get

$$
\begin{gathered}
I^{\prime}(t) \geq(-\mu-r) I(t), \quad t \neq n T, \\
I\left(t^{+}\right)=I(t), \quad t=n T,
\end{gathered}
$$

for $t \in\left[t^{*}, t^{*}+n_{2} T\right]$. Integrating (71) on $\left[t^{*}, t^{*}+n_{2} T\right]$, we have

$$
I\left(t^{*}+n_{2} T\right) \geq m_{4} e^{(-\mu-r) n_{2} T}
$$

Thus, we have

$$
I\left(t^{*}+T^{\prime}\right) \geq m_{4} e^{(-\mu-r) n_{2} T} e^{n_{3} \omega}>m_{4},
$$

which is a contradiction.

Let $\tilde{t}=\inf _{t>t^{*}}\left\{t \quad \mid I(t)>m_{4}\right\}$, and then for $t \in$ $\left(t^{*}, \widetilde{t}\right), I(t) \leq m_{4}$ and $I(\widetilde{t})=m_{4}$, since $I(t)$ is continuous and $I\left(t^{+}\right)=I(t)$ when $t=n T$. For $t \in\left(t^{*}, \widetilde{t}\right)$, suppose $\tilde{t} \in\left(t^{*}+(k-1) T, t^{*}+k T\right], k \in Z_{+}, k \leq n_{2}+n_{3}$; from (71), we have

$$
I(t) \geq I\left(t^{*}\right) e^{(-\mu-r)\left(t-t^{*}\right)} \geq m_{4} e^{\left(n_{2}+n_{3}\right)(-\mu-r) T} .
$$

Let $m_{2}^{\prime}=m_{4} e^{\left(n_{2}+n_{3}\right)(-\mu-r) T}$, hence; we have $I(t) \geq m_{2}^{\prime}$ for $t \in$ $\left(t^{*}, \widetilde{t}\right)$. For $t>\widetilde{t}$, the same arguments can be continued since $I(\widetilde{t}) \geq m_{4}$.

Case (II). $t^{*} \neq n T, n \in Z_{+}$. Then $I(t) \geq m_{4}$ for $t \in\left[t_{1}, t^{*}\right]$ and $I\left(t^{*}\right)=m_{4}$; suppose $t^{*} \in\left(n_{4} T,\left(n_{4}+1\right) T\right), n_{4} \in Z_{+}$. There are two possible cases for $t \in\left(t^{*},\left(n_{4}+1\right) T\right), n_{4} \in Z_{+}$.

Case (IIa). $I(t) \leq m_{4}$ for all $t \in\left(t^{*},\left(n_{4}+1\right) T\right)$. We claim that there must be a $t_{2}^{\prime} \in\left[\left(n_{4}+1\right) T,\left(n_{4}+1\right) T+T^{\prime}\right]$ such that $I\left(t_{2}^{\prime}\right)>m_{4}$. Otherwise, consider (62) with $m\left(\left(n_{4}+1\right) T^{+}\right)=$ $S\left(\left(n_{4}+1\right) T^{+}\right)$; we have

$$
\left|m(t)-m^{*}(t)\right| \leq\left(1+m^{*}\right) e^{-\mu\left(t-\left(n_{4}+1\right) T\right)}
$$

for $t \in(n T,(n+1) T]$ and $n_{4}+1 \leq n \leq n_{4}+1+n_{2}+n_{3}$. By a similar argument as in step II case (I), we get

$$
I\left(\left(n_{4}+1+n_{2}+n_{3}\right) T\right) \geq I\left(\left(n_{4}+1+n_{2}\right) T\right) e^{n_{3} \omega} .
$$

Since $I(t) \leq m_{4}$ for $t \in\left(t^{*},\left(n_{4}+1\right) T\right),(71)$ holds on $\left[t^{*},\left(n_{4}+\right.\right.$ $\left.\left.1+n_{2}\right) T\right]$, so we have

$$
I\left(\left(n_{4}+1+n_{2}\right) T\right) \geq m_{4} e^{\left(n_{2}+1\right)(-\mu-r) T} .
$$

Thus

$$
I\left(\left(n_{4}+1+n_{2}+n_{3}\right) T\right) \geq m_{4} e^{\left(n_{2}+1\right)(-\mu-r) T} e^{n_{3} \omega}>m_{4},
$$

which is a contradiction. Let $\bar{t}=\inf _{t>t^{*}}\left\{t \mid I(t)>m_{4}\right\}$, and then for $\in\left(t^{*}, \bar{t}\right), I(t) \leq m_{4}$ and $I(\bar{t})=m_{4}$. For $t \in\left(t^{*}, \bar{t}\right)$, suppose $\bar{t} \in\left(n_{4} T+\left(k^{\prime}-1\right) T, n_{4} T+k^{\prime} T\right], k^{\prime} \in Z_{+}, k^{\prime} \leq$ $1+n_{2}+n_{3}$, we have

$$
I(t) \geq m_{4} e^{\left(1+n_{2}+n_{3}\right)(-\mu-r) T} .
$$

Let $m_{2}=m_{4} e^{\left(1+n_{2}+n_{3}\right)(-\mu-r) T}<m_{2}^{\prime}$, so $I(t) \geq m_{2}$ for $t \in\left(t^{*}, \bar{t}\right)$. For $t>\bar{t}$, the same arguments can be continued since $I(\bar{t}) \geq$ $m_{4}$.

Case (IIb). There exists a $t \in\left(t^{*},\left(n_{4}+1\right) T\right)$ such that $I(t)>m_{4}$. Let $t^{* *}=\inf _{t>t^{*}}\left\{t \mid I(t)>m_{4}\right\}$, and then for $t \in\left(t^{*}, t^{* *}\right), I(t) \leq m_{4}$ and $I\left(t^{* *}\right)=m_{4}$. For $t \in\left(t^{*}, t^{* *}\right)$, integrating (71) on $\left(t^{*}, t^{* *}\right)$, we have

$$
I(t) \geq I\left(t^{*}\right) e^{(-\mu-r)\left(t-t^{*}\right)} \geq m_{4} e^{(-\mu-r) T}>m_{2} .
$$

So, $I(t) \geq m_{2}$ for $t \in\left(t^{*}, t^{* *}\right)$. Since $I\left(t^{* *}\right) \geq m_{4}$, for $t>t^{* *}$, the same arguments can be continued.

Hence, $I(t) \geq m_{2}$ for all $t \geq t_{1}$. The proof is completed.

\section{Numerical Analysis and Conclusion}

To verify the theoretical results obtained in this paper, we will give some numerical simulations. We consider the hypothetical set of parameter values as $\Lambda=0.2, \mu=0.2$, $\beta=0.1, r=0.3$, and $a=0.1$ with $\left(S_{0}, I_{0}, Z_{0}\right)=(0.7,0.3,0.3)$. By calculation, we know that $R=0.2<1$, and according to Theorem 5, and we know that the disease-free equilibrium $E_{0}(1,0,1)$ of system $(7)$ is locally asymptotically stable for this case (see Figure 1). We set the hypothetical set of parameter values as $\Lambda=1.8, \mu=0.2, \beta=0.08, r=0.3, a=0.1$ with $\left(S_{0}, I_{0}, Z_{0}\right)=(0.7,0.3,0.3)$. By calculation, we know that $R=1.44>1$, and according to Theorem 6 , we know that the endemic equilibrium $E^{*}(6.25,1.1,6.25)$ of system (7) is locally asymptotically stable for this case (see Figure 2 ).

On the other hand, we consider the hypothetical set of parameter values as $\Lambda=1, \mu=0.2, \beta=0.5, r=0.6, a=3$, and $T=1$ with $\left(S_{0}, I_{0}, Z_{0}\right)=(0.7,0.3,0.3)$. If $\delta=0.8$, by calculation, we know that $\mathfrak{R}=0.4155<1$. According to Theorem 9, we know that the "infection-free" periodic solution of system (9) is globally asymptotically stable for this case (see Figure 3). We can explain this in the epidemiology that if we take such a strategy by improving vaccination 


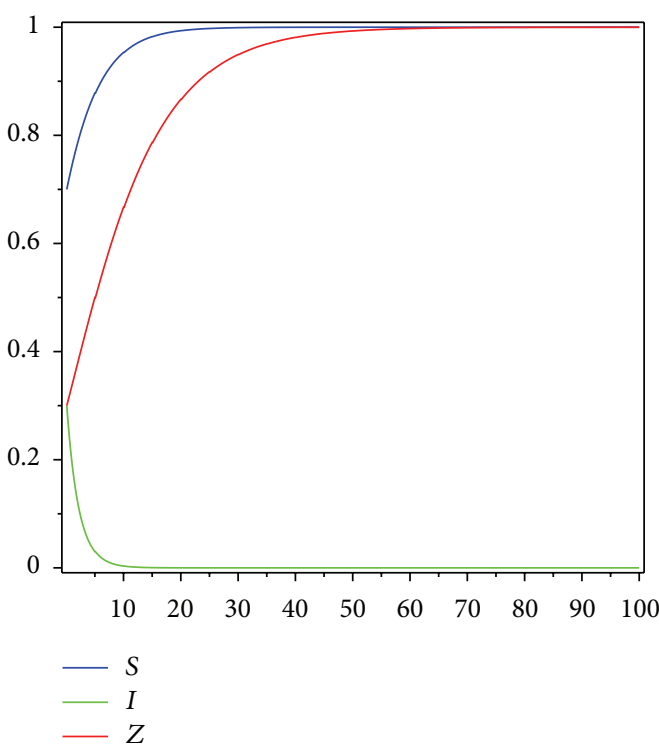

(a) Time series of $S(t), I(t), Z(t)$

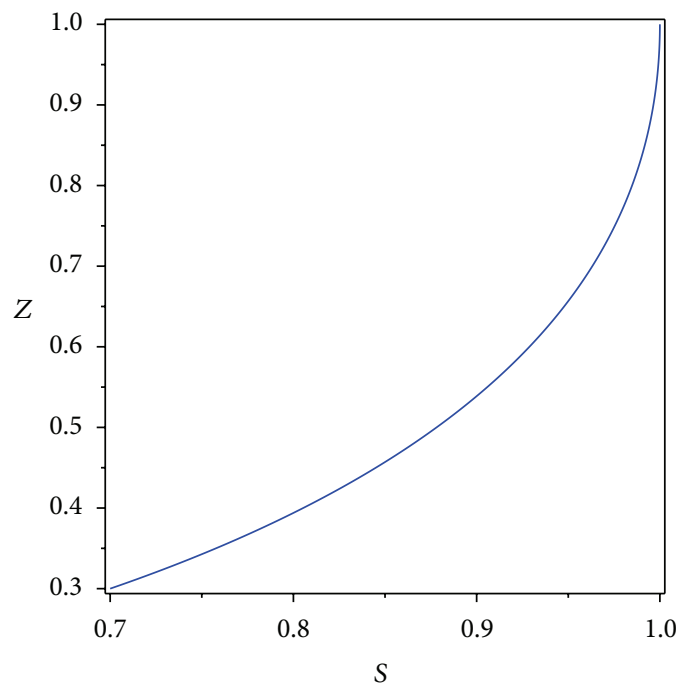

(c) Phase portrait of $S(t), Z(t)$

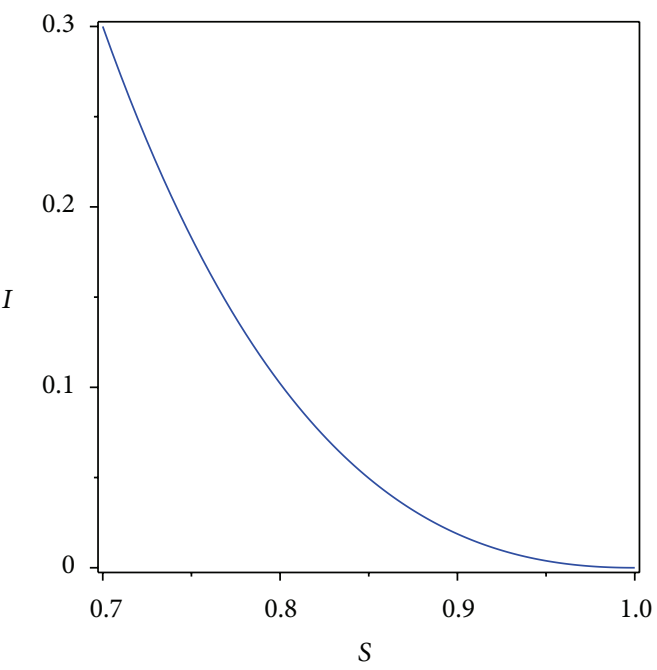

(b) Phase portrait of $S(t), I(t)$

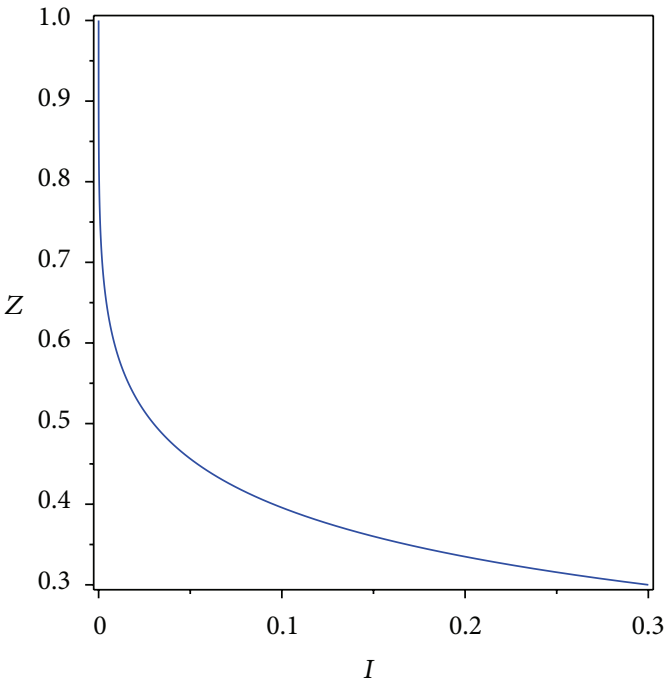

(d) Phase portrait of $I(t), Z(t)$

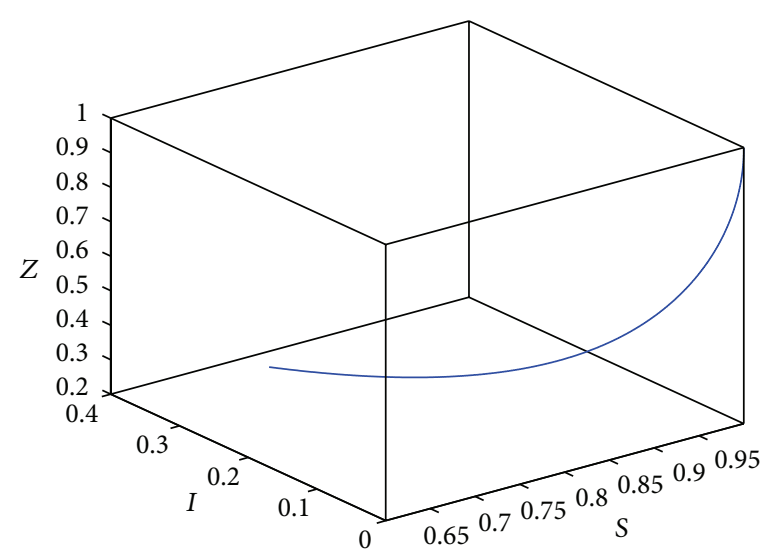

(e) Phase portrait of $S(t), I(t), Z(t)$

FIGURE 1: Illustration of basic behavior of system (7) on the threshold values $R=0.2<1$, where $\Lambda=0.2, \mu=0.2, \beta=0.1, r=0.3, a=0.1$, the initial value is $\left(S_{0}, I_{0}, Z_{0}\right)=(0.7,0.3,0.3)$, and $E_{0}=(1,0,1)$. 


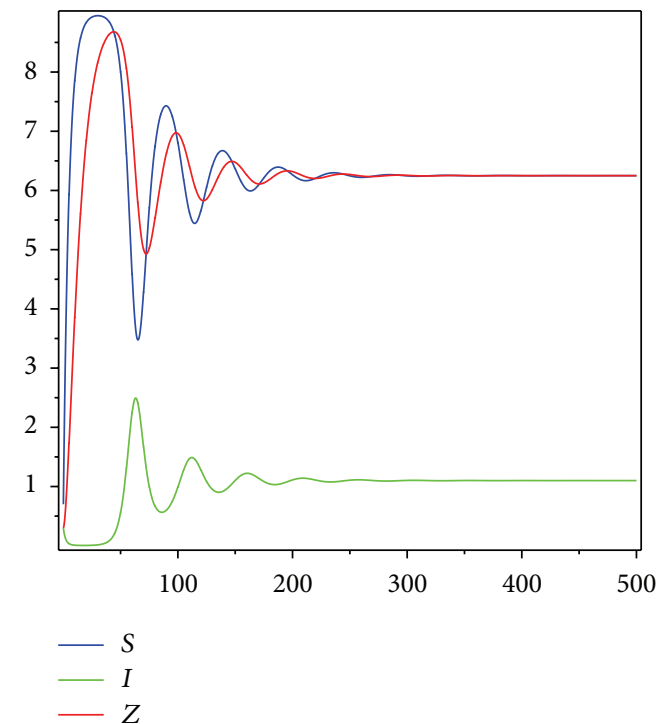

(a) Time series of $S(t), I(t), Z(t)$

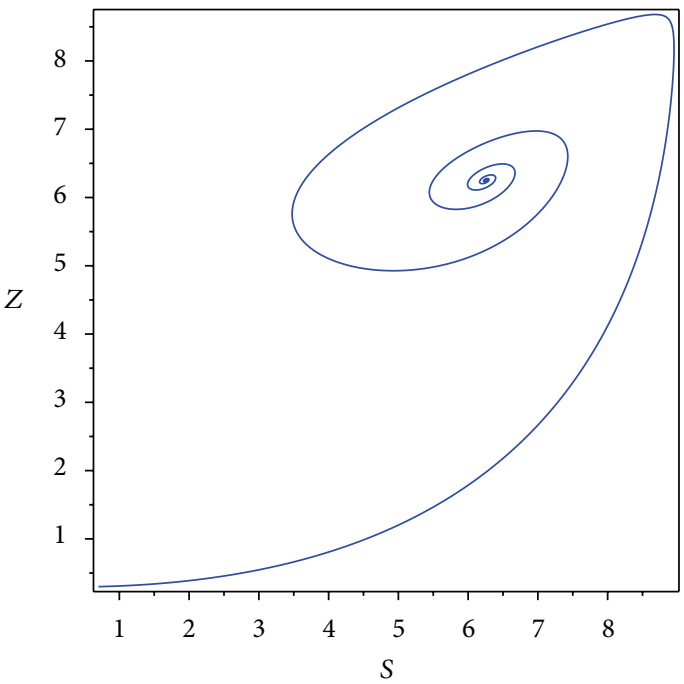

(c) Phase portrait of $S(t), Z(t)$

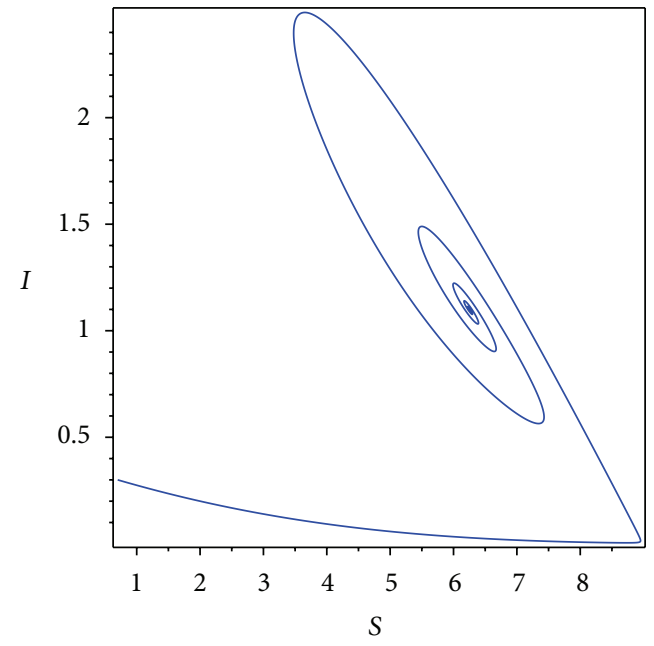

(b) Phase portrait of $S(t), I(t)$

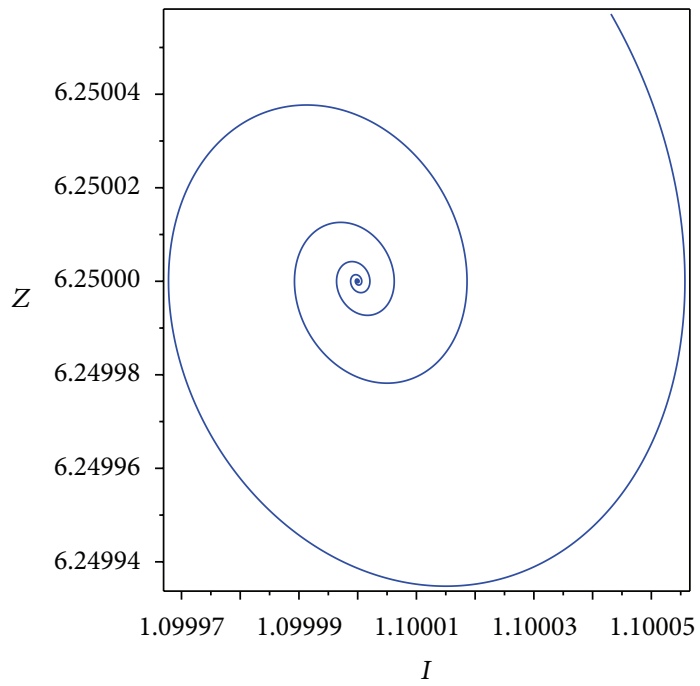

(d) Phase portrait of $I(t), Z(t)$

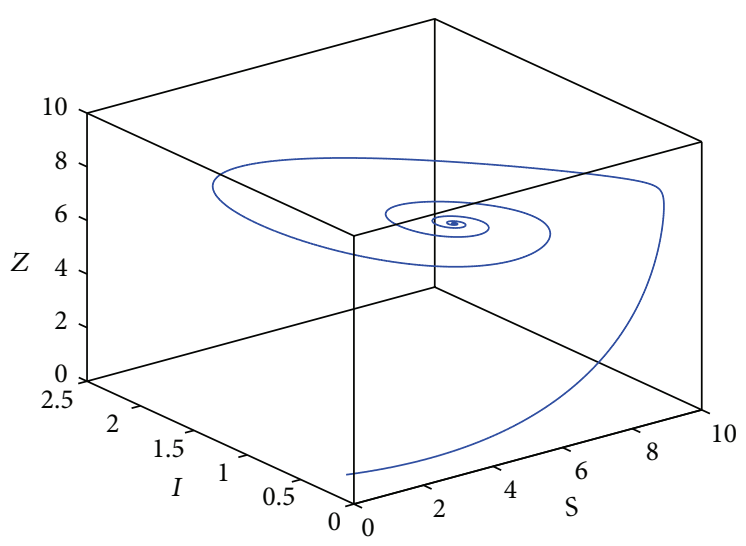

(e) Phase portrait of $S(t), I(t), Z(t)$

FIGURE 2: Illustration of basic behavior of system (7) on the threshold values $R=1.44>1$, where $\Lambda=1.8, \mu=0.2, \beta=0.08, r=0.3, a=0.1$, the initial value is $\left(S_{0}, I_{0}, Z_{0}\right)=(0.7,0.3,0.3)$, and $E^{*}=(6.25,1.1,6.25)$. 


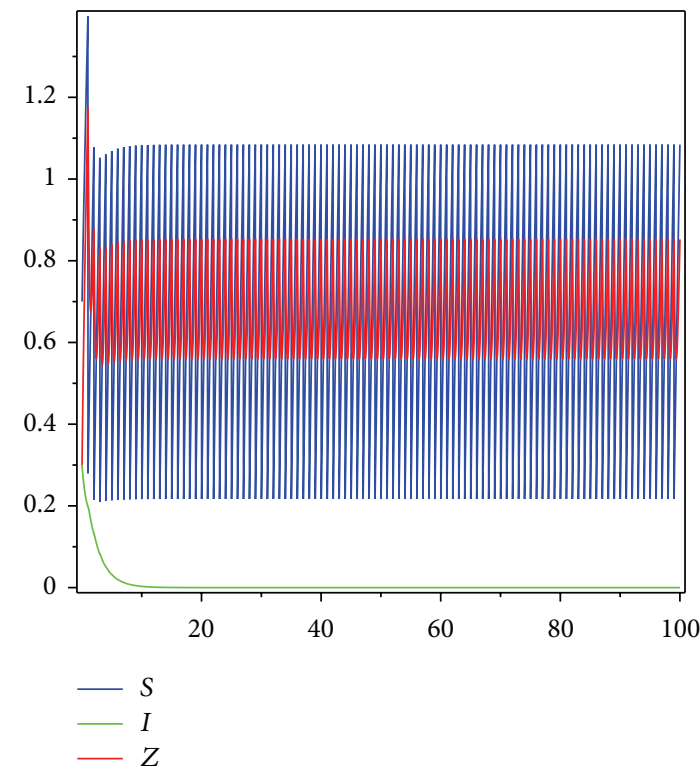

(a) Time series of $S(t), I(t), Z(t)$

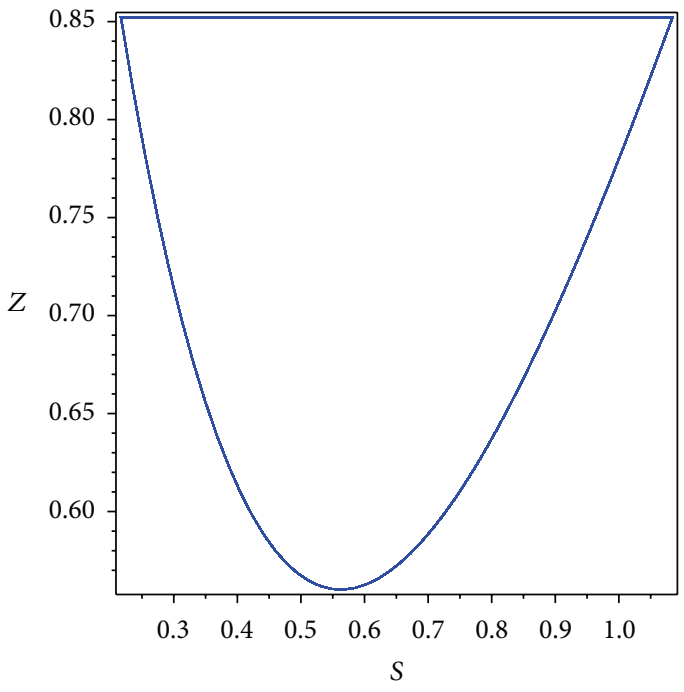

(c) Phase portrait of $S(t), Z(t)$

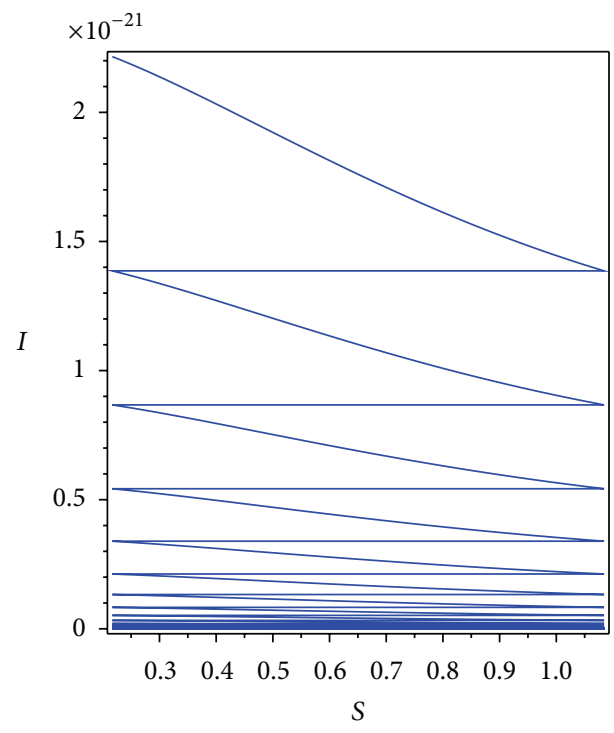

(b) Phase portrait of $S(t), I(t)$

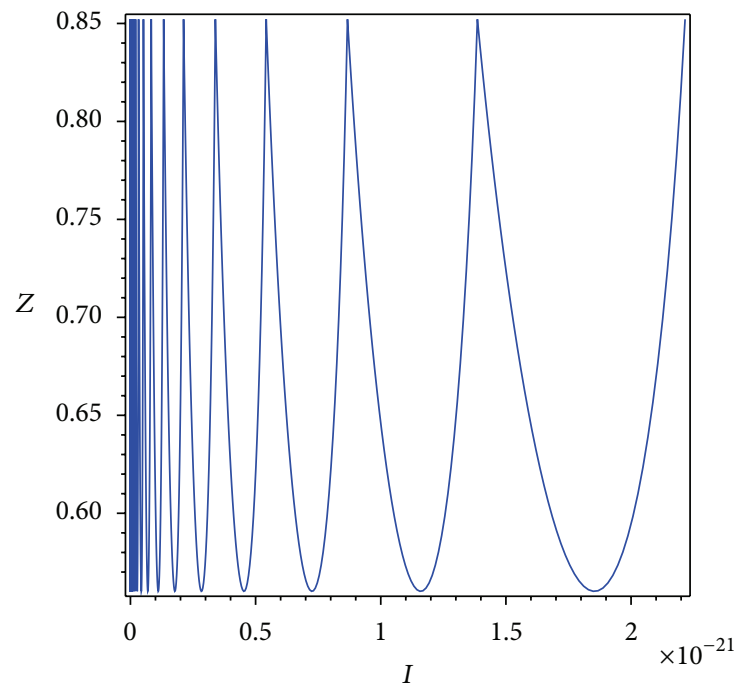

(d) Phase portrait of $I(t), Z(t)$

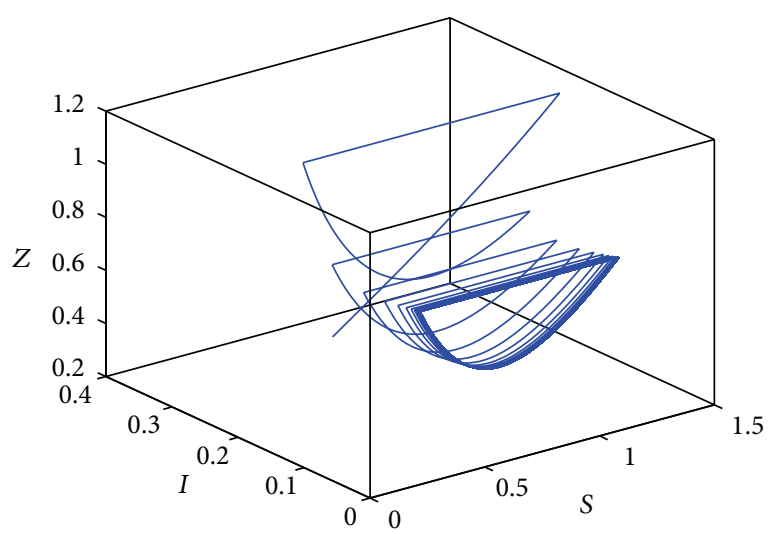

(e) Phase portrait of $S(t), I(t), Z(t)$

Figure 3: Illustration of basic behavior of system (9) on the threshold values $\Re=0.4155<1$, where $\Lambda=1, \mu=0.2, \beta=0.5, r=0.6, a=$ $3, T=1$, and $\delta=0.8$. 


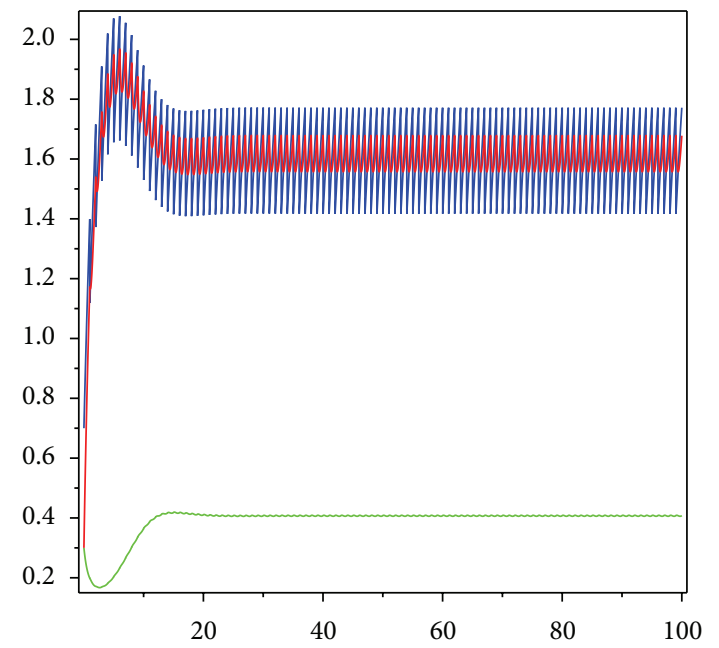

$-S$

$-I$
$-Z$

(a) Time series of $S(t), I(t), Z(t)$

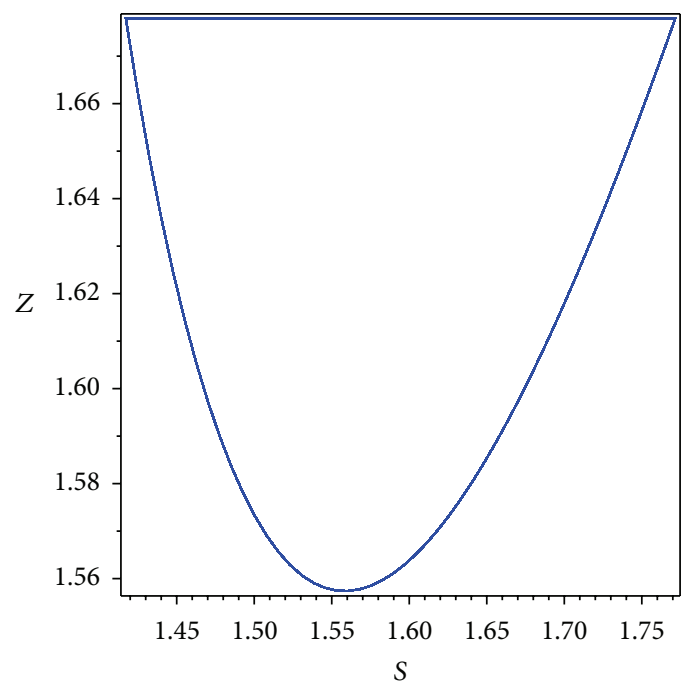

(c) Phase portrait of $S(t), Z(t)$

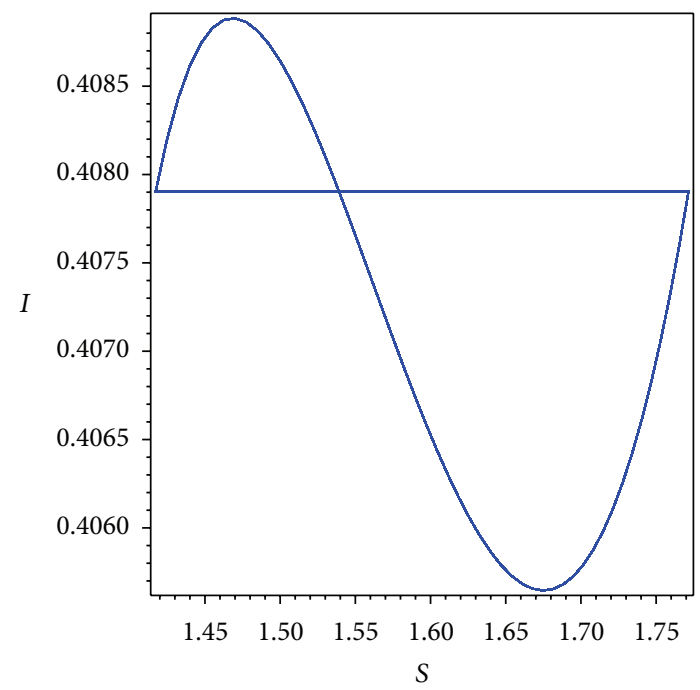

(b) Phase portrait of $S(t), I(t)$

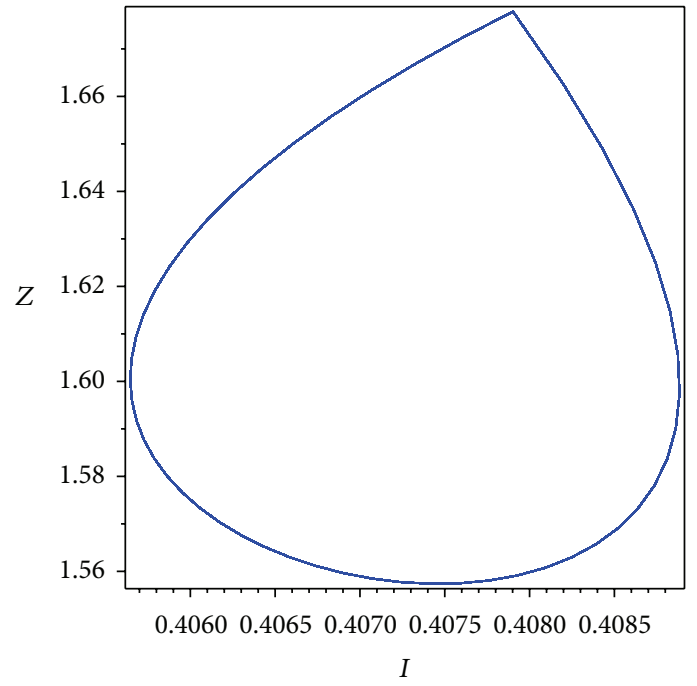

(d) Phase portrait of $I(t), Z(t)$

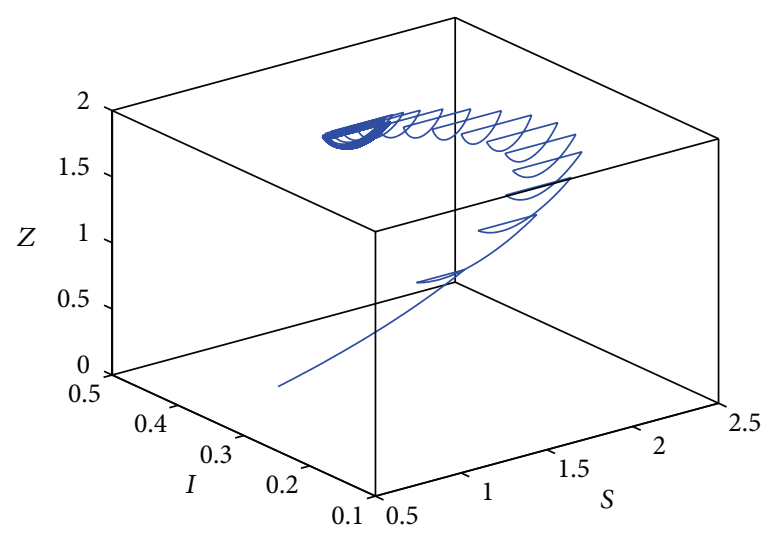

(e) Phase portrait of $S(t), I(t), Z(t)$

FIGURE 4: Illustration of basic behavior of system (9) on the threshold values $\Re=1.4831>1$, where $\Lambda=1, \mu=0.2, \beta=0.5, r=0.6, a=$ $3, T=1$, and $\delta=0.2$. 
proportion of susceptible persons in practice, as a result, the infectious population vanishes; that is, diseases eliminate. Figures 3(b), 3(c), 3(d), and 3(e) show the "infection-free" periodic solution under $\mathfrak{R}=0.4155<1$. In contrast, if we decrease vaccination proportion of susceptible persons to 0.2 , the diseases will be permanent (in this case $\mathfrak{R}=1.4831$.) (see Figure 4). Comparing Figure 1 with Figure 3 and Figure 2 with Figure 4, we find that the impulse vaccination proportion of susceptible persons has played a very important role in the actual epidemic prevention.

In this paper, a SIR epidemic model with distributed delay is proposed. The dynamics behavior of the model without vaccination or under impulsive vaccination is studied, respectively. By using the Jacobian matrix, the stability of the equilibrium points of the system without vaccination is analyzed and by using Floquet's theorem, small-amplitude perturbation skills and comparison theorem the sufficient conditions of globally asymptotic stability of "infection-free" periodic solution and the permanence of the model under impulsive vaccination are obtained. Lastly, we give some numerical simulation to illustrate our main conclusions. We think our mathematical results would be helpful in diseases control.

\section{Appendix}

\section{The Second Additive Compound Matrix}

Let $A=\left(a_{i j}\right)$ be a $3 \times 3$ matrix. Then its second additive compound matrix is as follows:

$$
A^{[2]}=\left(\begin{array}{ccc}
a_{11}+a_{22} & a_{23} & -a_{13} \\
a_{32} & a_{11}+a_{33} & a_{12} \\
-a_{31} & a_{21} & a_{22}+a_{33}
\end{array}\right) .
$$

Proposition. Let $\sigma A=\lambda_{i}: i=1,2,3$ be the spectrum of $A$. Then the spectrum of $A^{[2]}$ is $\sigma A^{[2]}=\lambda_{i}+\lambda_{j}: 1 \leq i<j \leq 3$.

\section{Acknowledgments}

The authors would like to thank the referees and the editor for their careful reading of the paper and many valuable comments and suggestions that greatly improved the presentation of this paper. This work is supported by Shandong Provincial Natural Science Foundation, China (no. ZR2012AM012), a Project of Shandong Province Higher Educational Science and Technology Program, China (no. J13LI05), and the SDUST Research Fund (no. 2011KYTD105).

\section{References}

[1] W. Kermack and A. G. McKendrick, "Contributions to the mathematical theory of epidemics," Proceedings of the Royal Society A, vol. 115, pp. 700-721, 1927.

[2] E. Beretta and Y. Takeuchi, "Global stability of an SIR epidemic model with time delays," Journal of Mathematical Biology, vol. 33, no. 3, pp. 250-260, 1995.

[3] E. Beretta and Y. Takeuchi, "Convergence results in SIR epidemic models with varying population sizes," Nonlinear
Analysi: Theory, Methods \& Applications, vol. 28, no. 12, pp. 1909-1921, 1997.

[4] K. L. Cooke, "Stability analysis for a vector disease model," The Rocky Mountain Journal of Mathematics, vol. 9, no. 1, pp. 31-42, 1979.

[5] W. M. Liu, S. A. Levin, and Y. Iwasa, "Influence of nonlinear incidence rates upon the behavior of SIRS epidemiological models," Journal of Mathematical Biology, vol. 23, no. 2, pp. 187204, 1986.

[6] M. Y. Li, J. R. Graef, L. Wang, and J. Karsai, "Global dynamics of a SEIR model with varying total population size," Mathematical Biosciences, vol. 160, no. 2, pp. 191-213, 1999.

[7] W. B. Ma, Y. Takeuchi, T. Hara, and E. Beretta, "Permanence of an SIR epidemic model with distributed time delays," The Tohoku Mathematical Journal, vol. 54, no. 4, pp. 581-591, 2002.

[8] W. B. Ma, M. Song, and Y. Takeuchi, "Global stability of an SIR epidemic model with time delay," Applied Mathematics Letters, vol. 17, no. 10, pp. 1141-1145, 2004.

[9] P. Yongzhen, L. Shuping, L. Changguo, and S. Z. Chen, "The effect of constant and pulse vaccination on an SIR epidemic model with infectious period," Applied Mathematical Modelling, vol. 35, no. 8, pp. 3866-3878, 2011.

[10] M. Song, W. B. Ma, and Y. Takeuchi, "Permanence of a delayed SIR epidemic model with density dependent birth rate," Journal of Computational and Applied Mathematics, vol. 201, no. 2, pp. 389-394, 2007.

[11] B. Shulgin, L. Stone, and Z. Agur, "Pulse vaccination strategy in the SIR epidemic model," Bulletin of Mathematical Biology, vol. 60, no. 6, pp. 1123-1148, 1998.

[12] Y. Takeuchi, W. B. Ma, and E. Beretta, "Global asymptotic properties of a delay SIR epidemic model with finite incubation times," Nonlinear Analysis: Theory, Methods \& Applications, vol. 42, pp. 931-947, 2000.

[13] Y. N. Xiao, L. S. Chen, and F. ven den Bosch, "Dynamical behavior for a stage-structured SIR infectious disease model," Nonlinear Analysis: Real World Applications, vol. 3, no. 2, pp. 175-190, 2002.

[14] H. Zhang, L. Chen, and J. J. Nieto, "A delayed epidemic model with stage-structure and pulses for pest management strategy," Nonlinear Analysis: Real World Applications, vol. 9, no. 4, pp. 1714-1726, 2008.

[15] T. Zhang and Z. Teng, "Global behavior and permanence of SIRS epidemic model with time delay," Nonlinear Analysis: Real World Applications, vol. 9, no. 4, pp. 1409-1424, 2008.

[16] T. L. Zhang and Z. D. Teng, "Permanence and extinction for a nonautonomous SIRS epidemic model with time delay," Applied Mathematical Modelling, vol. 33, no. 2, pp. 1058-1071, 2009.

[17] T. Q. Zhang, X. Z. Meng, T. H. Zhang, and Y. Song, "Global dynamics for a new high-dimensional SIR model with distributed delay," Applied Mathematics and Computation, vol. 218, no. 24, pp. 11806-11819, 2012.

[18] K. M. Charlton, S. Nadin-Davis, G. A. Casey, and A. I. Wandeler, "The long incubation period in rabies: delayed progression of infection in muscle at the site of exposure," Acta Neuropathologica, vol. 94, no. 1, pp. 73-77, 1997.

[19] D. B. Fishbein, "Rabies in humans," in The Natural History of Rabies, G. M. Baer, Ed., pp. 519-549, CRC Press, Boca Raton, Fla, USA, 2nd edition, 1991.

[20] M. Fekadu, "Canine rabies," in The Natural History of Rabies, G. M. Baer, Ed., pp. 267-278, CRC Press, Boca Raton, Fla, USA, 2nd edition, 1992. 
[21] P. J. Grattan-Smith, W. J. O’Regan, P. S. J. Ellis, S. J. O’Flaherty, P. B. McIntyre, and C. J. Barnes, "Rabies: a second Australian case, with a long incubation period," Medical Journal of Australia, vol. 156, no. 9, pp. 651-654, 1992.

[22] H.-F. Huo and Z.-P. Ma, "Dynamics of a delayed epidemic model with non-monotonic incidence rate," Communications in Nonlinear Science and Numerical Simulation, vol. 15, no. 2, pp. 459-468, 2010.

[23] C. A. de Quadros, J. K. Andrus, J.-M. Olive et al., "Eradication of poliomyelitis: progress in the Americas," Pediatric Infectious Disease Journal, vol. 10, no. 3, pp. 222-229, 1991.

[24] M. de la Sen, A. Ibeas, and S. Alonso-Quesada, "On vaccination controls for the SEIR epidemic model," Communications in Nonlinear Science and Numerical Simulation, vol. 17, no. 6, pp. 2637-2658, 2012.

[25] M. Ramsay, N. Gay, E. Miller et al., "The epidemiology of measles in England and Wales: rationale for the 1994 national vaccination campaign," Communicable Disease Report, vol. 4, no. 12, pp. R141-R146, 1994.

[26] A. B. Sabin, "Measles, killer of millions in developing countries: strategy for rapid elimination and continuing control," European Journal of Epidemiology, vol. 7, no. 1, pp. 1-22, 1991.

[27] D. Bainov and P. Simeonov, System With Impulsive Effect: Stability, Theory and Applications, John Wiley \& Sons, New York, NY, USA, 1989.

[28] S. J. Gao, L. S. Chen, J. J. Nieto, and A. Torres, "Analysis of a delayed epidemic model with pulse vaccination and saturation incidence," Vaccine, vol. 24, no. 35-36, pp. 6037-6045, 2006.

[29] B. Liu, Z. D. Teng, and W. B. Liu, "Dynamic behaviors of the periodic Lotka-Volterra competing system with impulsive perturbations," Chaos, Solitons and Fractals, vol. 31, no. 2, pp. 356-370, 2007.

[30] Z. X. Li, Z. Zhao, and L. S. Chen, "Bifurcation of a three molecular saturated reaction with impulsive input," Nonlinear Analysis: Real World Applications, vol. 12, no. 4, pp. 2016-2030, 2011.

[31] V. Lakshmikantham, D. D. Bainnov, and P. S. Simeonov, Theory of Impulsive Differential Equations, World Scientific, Singapore,, 1989.

[32] J. J. Nieto and R. Rodríguez-López, "Hybrid metric dynamical systems with impulses," Nonlinear Analysis: Theory, Methods \& Applicationss, vol. 64, no. 2, pp. 368-380, 2006.

[33] J. J. Nieto and R. Rodríguez-López, "New comparison results for impulsive integro-differential equations and applications," Journal of Mathematical Analysis and Applications, vol. 328, no. 2, pp. 1343-1368, 2007.

[34] X. Y. Song and Y. F. Li, "Dynamic behaviors of the periodic predator-prey model with modified Leslie-Gower Holling-type II schemes and impulsive effect," Nonlinear Analysis: Real World Applications, vol. 9, no. 1, pp. 64-79, 2008.

[35] S. J. Gao, Z. D. Teng, and D. H. Xie, "Analysis of a delayed SIR epidemic model with pulse vaccination," Chaos, Solitons \& Fractals, vol. 40, no. 2, pp. 1004-1011, 2009.

[36] J. Hui and L.-S. Chen, "Impulsive vaccination of SIR epidemic models with nonlinear incidence rates," Discrete and Continuous Dynamical Systems B, vol. 4, no. 3, pp. 595-605, 2004.

[37] Z. H. Lu, X. B. Chi, and L. S. Chen, "The effect of constant and pulse vaccination on SIR epidemic model with horizontal and vertical transmission," Mathematical and Computer Modelling, vol. 36, no. 9-10, pp. 1039-1057, 2002.
[38] X. Z. Meng, L. S. Chen, and H. D. Cheng, “Two profitless delays for the SEIRS epidemic disease model with nonlinear incidence and pulse vaccination," Applied Mathematics and Computation, vol. 186, no. 1, pp. 516-529, 2007.

[39] X. Z. Meng and L. S. Chen, "The dynamics of a new SIR epidemic model concerning pulse vaccination strategy," Applied Mathematics and Computation, vol. 197, no. 2, pp. 582-597, 2008.

[40] A. D'Onofrio, "On pulse vaccination strategy in the SIR epidemic model with vertical transmission," Applied Mathematics Letters, vol. 18, no. 7, pp. 729-732, 2005.

[41] X. Y. Song, Y. Jiang, and H. M. Wei, "Analysis of a saturation incidence SVEIRS epidemic model with pulse and two time delays," Applied Mathematics and Computation, vol. 214, no. 2, pp. 381-390, 2009.

[42] C. Castillo-Chavez and B. J. Song, "Dynamical models of tuberculosis and their applications," Mathematical Biosciences and Engineering, vol. 1, no. 2, pp. 361-404, 2004.

[43] D. Baĭnov and P. Simeonov, Impulsive Differential Equations: Periodic Solutions and Applications, vol. 66 of Pitman Monographs and Surveys in Pure and Applied Mathematics, 1993. 


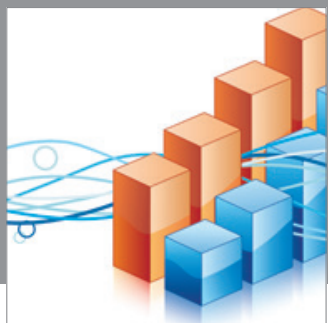

Advances in

Operations Research

mansans

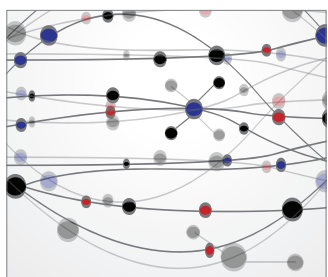

The Scientific World Journal
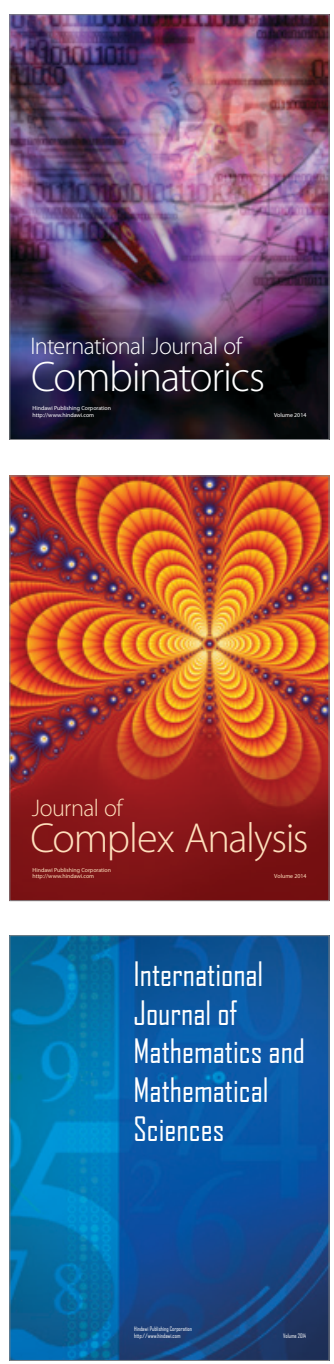
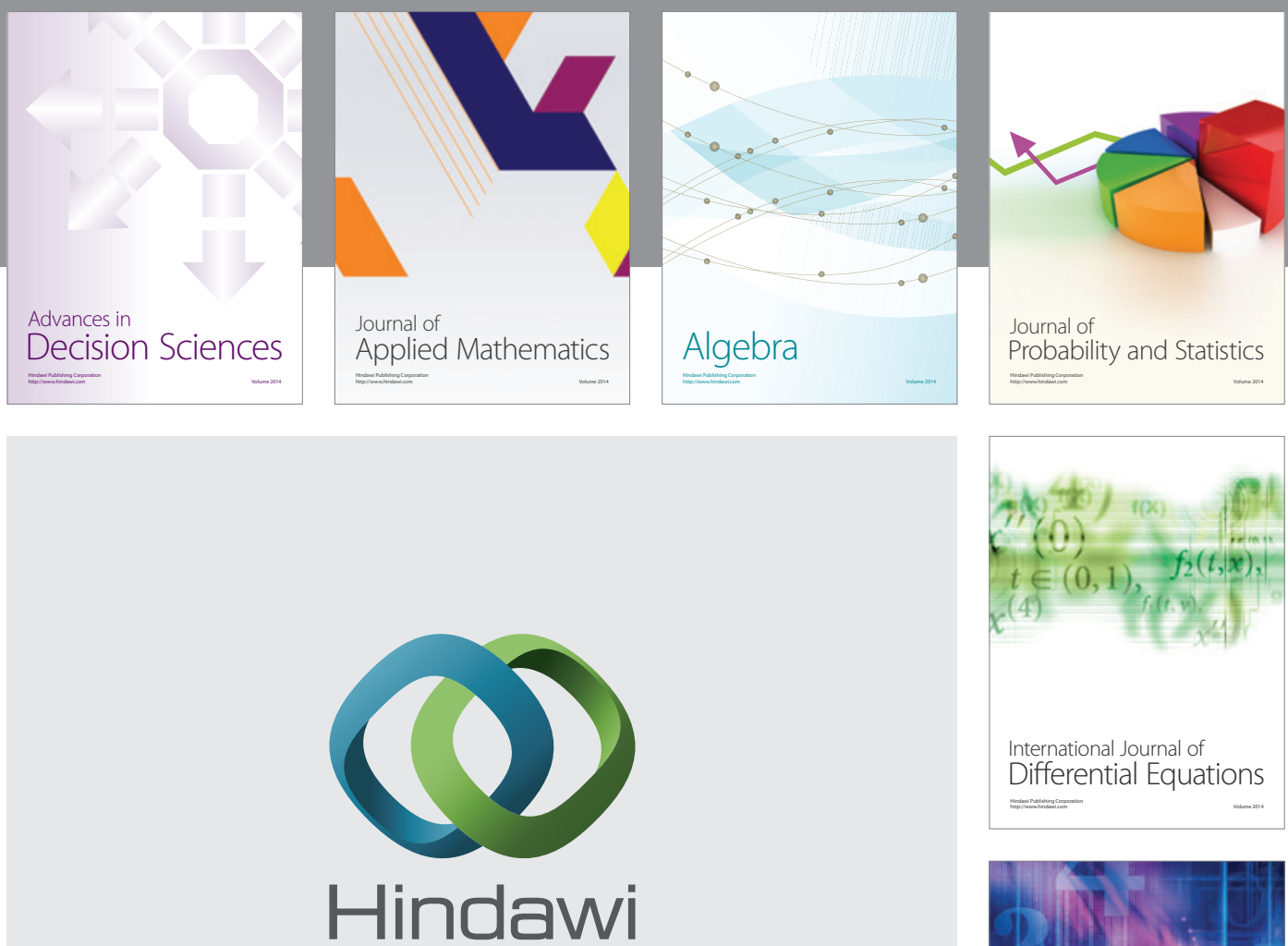

Submit your manuscripts at http://www.hindawi.com
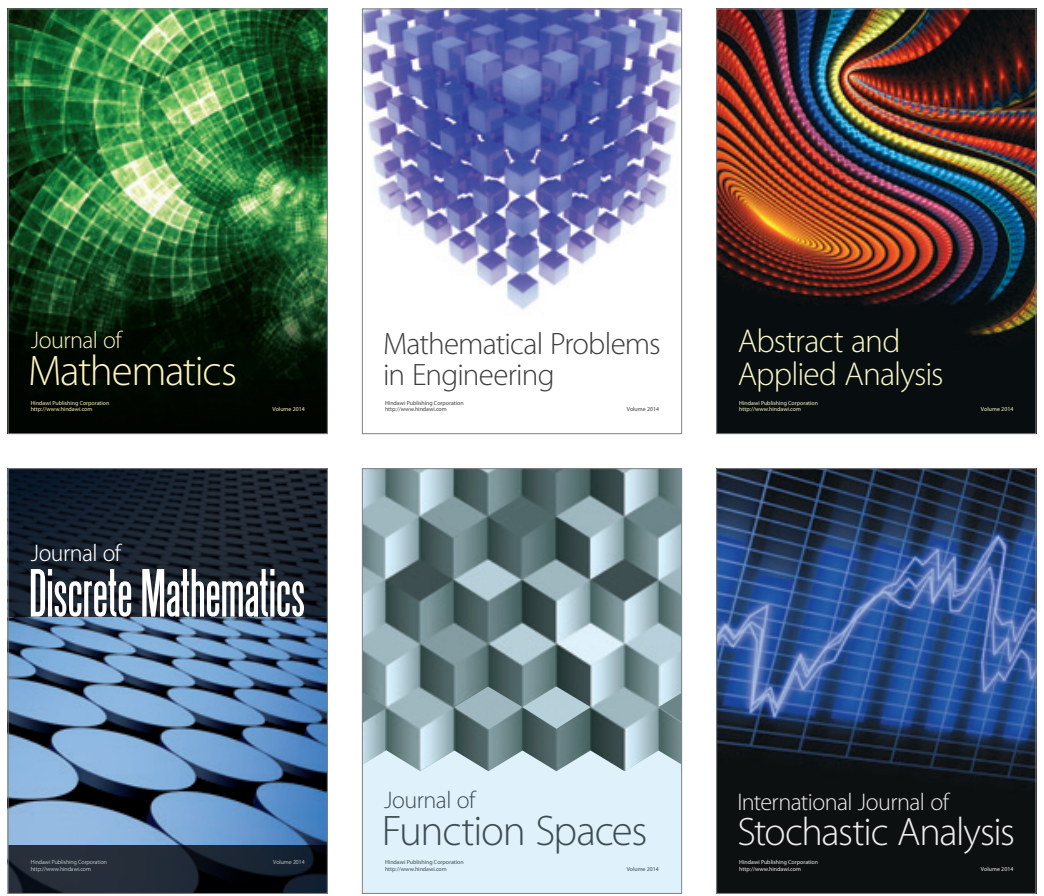

Journal of

Function Spaces

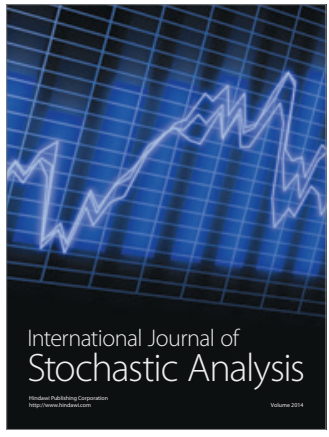

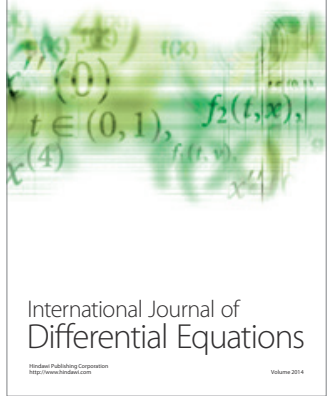
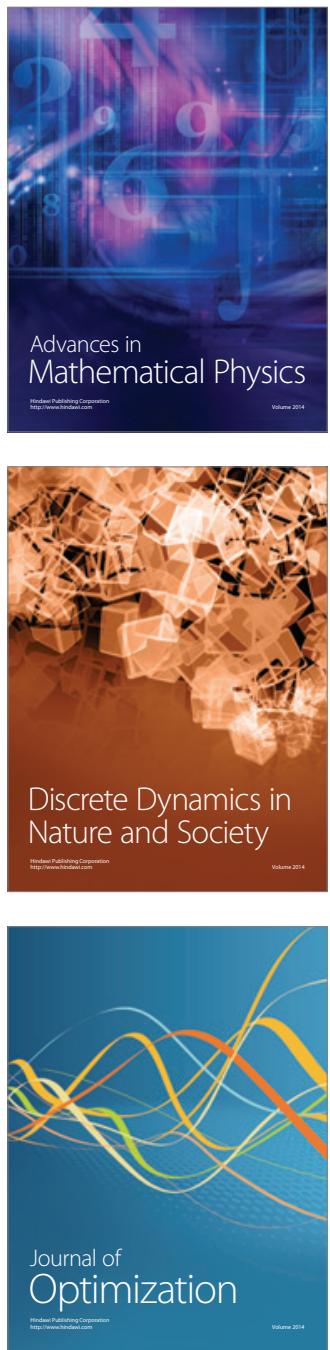